\title{
Efficient synthesis of novel benzo[b][1,8]naphthyridin-4(1H)-ones and pyrido[2,3-b]quinoxalin-4(1H)-ones from alkynones and primary amines
}

\author{
Viktor O. Iaroshenko a,b,*, Muhammad Zahid ${ }^{\mathrm{a}}$, Satenik Mkrtchyan ${ }^{\mathrm{a}}$, \\ Ashot Gevorgyan ${ }^{a}$, Kai Altenburger ${ }^{a}$, Ingo Knepper ${ }^{a}$, Alexander Villinger ${ }^{a}$, \\ Vyacheslav Ya. Sosnovskikh ${ }^{\mathrm{c}}$, Peter Langer ${ }^{\mathrm{a}, \mathrm{d}}$ \\ a Institut für Chemie, Universität Rostock, Albert Einstein Str. 3a, 18059 Rostock, Germany \\ ${ }^{\mathrm{b}}$ National Taras Shevchenko University, 62 Volodymyrska Str., 01033 Kyiv, Ukraine \\ ${ }^{\mathrm{c}}$ Department of Chemistry, Ural Federal University, 51 Lenina Ave., 620083 Ekaterinburg, Russia \\ ${ }^{\mathrm{d}}$ Leibniz-Institut für Katalyse an der Universität Rostock e.V., Albert Einstein Str. 29a, 18059 Rostock, Germany
}

\section{A R T I C L E I N F O}

\section{Article history:}

Received 17 November 2012

Accepted 4 January 2013

Available online 10 January 2013

Keywords:

o-Chlorohetaryl ynones

Amines

Palladium-catalyzed cyclization

Benzo[b][1,8]naphthyridin-4(1H)-ones

Pyrido[2,3-b]quinoxalin-4(1H)-ones

\begin{abstract}
A B S T R A C T
An efficient palladium-catalyzed cyclization of 0 -chlorohetaryl ynones with aliphatic and aromatic primary amines represents a simple access to a wide range of benzo[b][1,8]naphthyridin- $4(1 H)$-one and pyrido[2,3-b]quinoxalin-4(1H)-one derivatives in good to excellent yields.
\end{abstract}

(c) 2013 Elsevier Ltd. All rights reserved.

\section{Introduction}

Of the six isomeric pyridopyridines (diazanaphthalenes), 1,8naphthyridines are the most studied subclass because their skeleton is present in many compounds, which have been isolated from natural substances and exhibit various biological activities. They represent important lead structures in medicinal chemistry (treatment of various human diseases) and agricultural chemistry (use as pesticides). Among 1,8-naphthyridine derivatives, 6-fluoro-1,8-naphthyridin-4-ones, which are azaanalogs of fluoroquinolones, have attracted the most attention in the last 30 years. ${ }^{1}$ There are a number of drugs with 4-pyridone ring, such as enoxacin, ${ }^{2}$ a fluoronaphthyridone antibacterial agent showing the most broad and potent in vitro antibacterial activity, an excellent in vivo efficacy on systemic infections, and a weak acute toxicity, and trovafloxacin, ${ }^{3}$ which is a broad spectrum antibiotic and inhibits the uncoiling of supercoiled DNA in various bacteria by blocking the activity of DNA gyrase and topoisomerase IV (Fig. 1). Its spectrum of activity includes aerobic gram-positive

\footnotetext{
* Corresponding author. Tel.: +49 381 4986410; fax: +49 381 49864112; e-mail addresses: viktor.iaroshenko@uni-rostock.de, iva108@googlemail.com (V.O. laroshenko).
}<smiles>CCn1cc(C(=O)O)c(=O)c2cc(F)c(N3CCNCC3)nc21</smiles>

Fig. 1. Important 1,8-naphthyridin-4(1H)-ones.

and gram-negative organisms as well as anaerobic pathogens, however, it was withdrawn from the market due to the risk of hepatotoxicity.

Given the broad utility of 1,8-naphthyridines in medicinal chemistry ${ }^{1}$ and continuing our research on the efficient synthesis of drug-like heteroannulated pyridines, ${ }^{4}$ we were interested in developing novel approaches, which would allow access to these interesting heterocyclic compounds. In previous study dealing with the domino amination/conjugate addition reaction of 1-(2chloropyridin-3-yl)prop-2-yn-1-ones with amines, Iaroshenko's 
group described the synthesis of 1,8 -naphthyridin-4(1H)-ones. ${ }^{5}$ Though this method of preparation of 1,8-naphthyridine core seems to be satisfactory, new routes starting from different materials are desirable for efficient structure modifications of these molecules. In connection with this, we decided to prepare benzo[ $b][1,8]$ naphthyridin-4(1H)-one and pyrido[2,3- $b]$ quinoxalin-4(1H)-one derivatives, commencing with quinoline and quinoxaline-based substrates. Earlier 2,5-diarylbenzo[b][1,8]naphthyridin-4(1H)-ones have been constructed from 3-acetyl-4arylquinolin-2(1H)-ones via the intermediates 3-cinnamoyl-4arylquinolin-2(1H)-ones, ${ }^{6}$ while pyrido[2,3-b]quinoxalin-4(1H)ones represent a novel heterocyclic system.

In this paper we report the successful realization of our goal with an efficient palladium-catalyzed tandem amination approach, which was developed in one pot to afford pyridoannulated quinolines and quinoxalines from easily accessible o-chlorohetaryl acetylenic ketones and primary amines.

\section{Results and discussion}

Based on previous experience of the Iaroshenko's group related to the development of new coupling reactions of pyridinyl alkynones $^{5}$ and alkynyl thiophenes, ${ }^{7}$ we envisaged that $1-(2-$ chloroquinolin-3-yl)-3-phenylprop-2-yn-1-one (2), which can be prepared by allowing a lithium acetylide to react with 2-chloro-3formylquinoline, followed by oxidation of the resulting 1-(2chloroquinolin-3-yl)-3-phenylprop-2-yn-1-ol (1) ${ }^{8}$ is suitable substrate for the synthesis of benzo[b][1,8]naphthyridin- $4(1 H)$-one derivatives 3 (Scheme 1). The requisite starting material, 2-chloro3-formylquinoline, was obtained by treatment of acetanilide with the Vilsmeier reagent $\left(\mathrm{DMF} / \mathrm{POCl}_{3}\right)$ according to the described procedure. $^{9}$<smiles>O=Cc1cc2ccccc2nc1Cl</smiles>

$$
3 h, \triangle \mid \mathrm{MnO}_{2} / \mathrm{CH}_{2} \mathrm{Cl}_{2}
$$<smiles>[R]N/C(=C\C(=O)c1cc2ccccc2nc1Cl)PC</smiles>

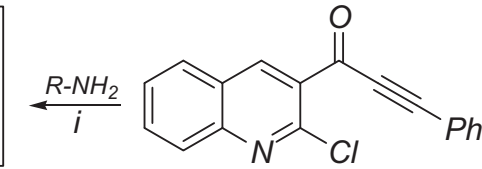

A $2(91 \%)$<smiles>[R]n1c(-c2ccccc2)cc(=O)c2cc3ccccc3nc21</smiles>

Scheme 1. Reaction conditions: (i) $\mathrm{Pd}\left(\mathrm{PPh}_{3}\right)_{4}, \mathrm{~K}_{2} \mathrm{CO}_{3}, \mathrm{DMF}, 170{ }^{\circ} \mathrm{C}, 3 \mathrm{~h}$ (for $\mathbf{3 a}-\mathbf{l}$ ); $\mathrm{Pd}\left(\mathrm{PPh}_{3}\right)_{4}, \mathrm{Cs}_{2} \mathrm{CO}_{3}$, toluene, $90{ }^{\circ} \mathrm{C}, 6 \mathrm{~h}$ (for $3 \mathbf{m}-\mathbf{u}$ ).

Then we examined the reactions of alkynone $\mathbf{2}$ with a range of commercially available aliphatic and aromatic amines. After the optimization of the reaction conditions with regard to the type of catalyst and solvent, we have found that the use of $\mathrm{Pd}\left(\mathrm{PPh}_{3}\right)_{4}$ ( $5 \mathrm{~mol} \%), 2$ equiv of $\mathrm{K}_{2} \mathrm{CO}_{3}$ as a base, and DMF as a solvent $\left(170{ }^{\circ} \mathrm{C}\right.$, $3 \mathrm{~h}$ ) was essential to get good yields $(46-87 \%)$ of benzo $[b][1,8]$
naphthyridin-4(1H)-ones $\mathbf{3 a - 1}$ from aliphatic amines. In the case of less nucleophilic aromatic amines, compounds $\mathbf{3 m}-\mathbf{u}$ were obtained with $\mathrm{Pd}\left(\mathrm{PPh}_{3}\right)_{4}$ (5 mol \%), $\mathrm{Cs}_{2} \mathrm{CO}_{3}$ (1.2 equiv) in toluene $\left(90^{\circ} \mathrm{C}, 6 \mathrm{~h}\right)$ in variable yields (29-77\%). When allylamine was used, product $3 \mathrm{~g}$ was obtained in $90 \%$ yield as a result of cleavage of the allyl group under the reaction conditions employed.

The progress of the reaction was monitored by TLC, and the results are summarized in Table 1 . It is important that a wide range of aromatic and aliphatic amines can effectively participate in the reaction with acetylenic ketone $\mathbf{2}$, providing a variety of benzofused 1,8-naphthyridines 3 with high purity after column chromatography. It can be observed that the process tolerates only electrondonating substituents (alkyl, alkoxy, diethylamino) on the aromatic amines. This is a palladium-catalyzed tandem reaction consisting of a sequential double $\mathrm{C}-\mathrm{N}$ bond formation to give benzo [b][1,8]naphthyridin-4(1H)-ones 3 from quinolinyl acetylenic ketone $\mathbf{2}$ and primary amines via intermediates $\mathbf{A}^{5,10}$ (Scheme 1). The structures of all products 3 were characterized by IR, ${ }^{1} \mathrm{H},{ }^{13} \mathrm{C}$ NMR spectral data as well as HRMS analysis. Furthermore, the structure of compound $\mathbf{3 f}$ was established by X-ray crystallographic analysis (Fig. 2). ${ }^{11}$

Table 1

1-Substituted 2-phenylbenzo[b][1,8]naphthyridin-4(1H)-ones 3a-u

\begin{tabular}{lll}
\hline $\mathbf{3}$ & $\mathrm{R}$ & Yield (\%) \\
\hline $\mathbf{a}$ & Cyclohexyl & 72 \\
$\mathbf{b}$ & Benzyl & 85 \\
$\mathbf{c}$ & p-Methoxybenzyl & 64 \\
$\mathbf{d}$ & Phenethyl & 46 \\
$\mathbf{e}$ & n-Heptyl & 65 \\
$\mathbf{f}$ & Cyclopropyl & 57 \\
$\mathbf{g}$ & $\mathrm{H}$ & 90 \\
$\mathbf{h}$ & 3-(4-Morpholino)propyl & 87 \\
$\mathbf{i}$ & HOCH $\mathrm{CH}_{2}$ & 72 \\
$\mathbf{j}$ & 3-Methoxybenzyl & 52 \\
$\mathbf{k}$ & 2-Chlorobenzyl & 86 \\
$\mathbf{1}$ & N-(3-Propyl)imidazole & 70 \\
$\mathbf{m}$ & 4-Methoxyphenyl & 73 \\
$\mathbf{n}$ & 3,4-Dimethoxyphenyl & 76 \\
$\mathbf{o}$ & 3,5-Dimethoxyphenyl & 64 \\
$\mathbf{p}$ & 3,4,5-Trimethoxyphenyl & 77 \\
$\mathbf{q}$ & 3,5-Dimethylphenyl & 58 \\
$\mathbf{r}$ & 2,4-Dimethoxyphenyl & 53 \\
$\mathbf{s}$ & 4-(Diethylamino)phenyl & 77 \\
$\mathbf{t}$ & 4-Ethylphenyl & 50 \\
$\mathbf{u}$ & 2-Fluorophenyl & 29 \\
\hline
\end{tabular}

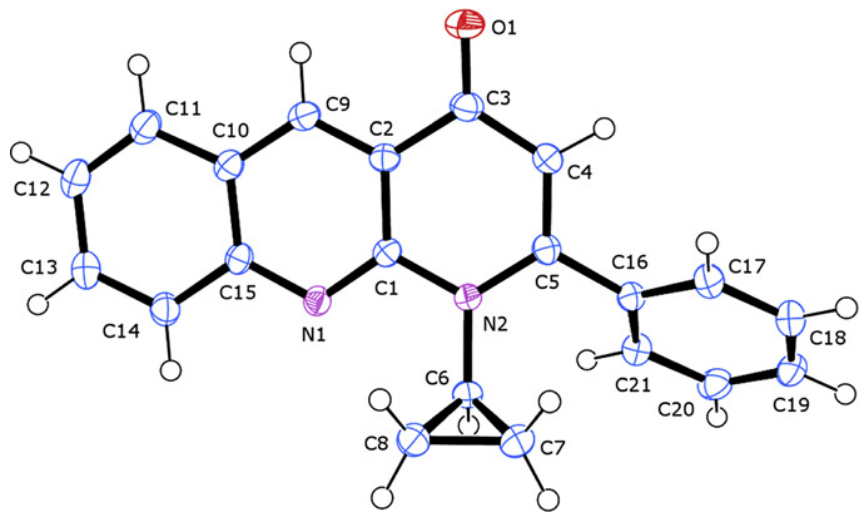

Fig. 2. Molecular structure of compound $\mathbf{3 f}$.

Next, we have obtained a series of 1-(3-chloroquinoxalin-2-yl) prop-2-yn-1-ones 5 by Sonogashira coupling of 3-chloroquinoxaline2-carbonyl chloride (4), obtained from 3-chloroquinoxaline-2carboxylic acid and thionyl chloride, ${ }^{12}$ with terminal alkynes $\left(\mathrm{R}^{\prime}=\mathrm{Ph}, 4-t-\mathrm{BuC}_{6} \mathrm{H}_{4}, n-\mathrm{C}_{8} \mathrm{H}_{17}\right)$ using $\mathrm{PdCl}_{2}\left(\mathrm{PPh}_{3}\right)_{2}$ in THF to give ketones $5 \mathbf{a}-\mathbf{c}$ in $35-58 \%$ yield. Reactions of these compounds with 
aliphatic and aromatic amines heated with or without $\mathrm{Pd}\left(\mathrm{PPh}_{3}\right)_{4}$ in the presence of $\mathrm{K}_{2} \mathrm{CO}_{3}$ in DMF produced pyrido[2,3-b]quinoxalin$4(1 H)$-ones $\mathbf{6 a}-\mathbf{n}$ (Scheme 2, Table 2). As illustrated in Table 2, alkynones $\mathbf{5 a}-\mathbf{c}$ readily reacted with various amines to give the corresponding products in moderate to high yields. While aromatic amines required the employment of a Pd catalyst (Methods B-D), reactions of aliphatic amines proceeded smoothly under catalyst free conditions (Method A). When $\mathrm{Pd}\left(\mathrm{PPh}_{3}\right)_{4}$ was used as catalyst and combined with rac-2,2'-bis(diphenylphosphino)-1,1'-binaphthyl (rac-BINAP) or 2-dicyclohexylphosphino-2'-(N,N-dimethylamino)biphenyl (DavePhos), the yields were improved slightly (compounds $\mathbf{6 c}, \mathbf{g}, \mathbf{h}$ ). The nature of the acetylene moiety has little effect on the yield of these reactions.

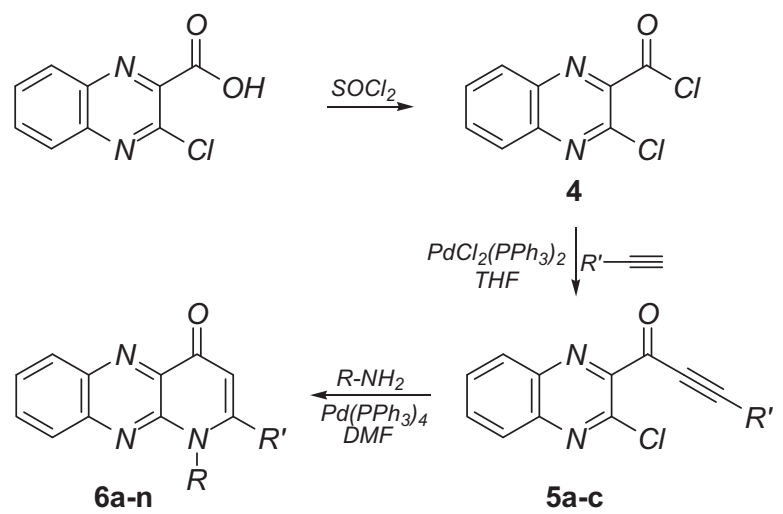

Scheme 2. Synthesis of pyrido[2,3-b]quinoxalin-4(1H)-ones $\mathbf{6 a - n}$.

Table 2

1,2-Disubstituted pyrido[2,3-b]quinoxalin-4(1H)-ones 6a-n

\begin{tabular}{llll}
\hline $\mathbf{6}$ & $\mathrm{R}^{\prime}$ & $\mathrm{R}$ & Yield $(\%)$ \\
\hline $\mathbf{a}$ & $\mathrm{Ph}$ & Cyclohexyl & $91,{ }^{\mathrm{a}} 90,{ }^{\mathrm{b}} 90^{\mathrm{c}}$ \\
$\mathbf{b}$ & $\mathrm{Ph}$ & $n$-Heptyl & $99,{ }^{\mathrm{a}} 90,{ }^{\mathrm{b}} 87^{\mathrm{d}}$ \\
$\mathbf{c}$ & $\mathrm{Ph}$ & $\mathrm{HOCH} \mathrm{CH}_{2}$ & $41,{ }^{\mathrm{a}} 53,{ }^{\mathrm{b}} 71^{\mathrm{c}}$ \\
$\mathbf{d}$ & $\mathrm{Ph}$ & $\mathrm{Bn}$ & $70^{\mathrm{a}}$ \\
$\mathbf{e}$ & $\mathrm{Ph}$ & Phenethyl & $77,{ }^{\mathrm{a}} 84^{\mathrm{b}}$ \\
$\mathbf{f}$ & $\mathrm{Ph}$ & $2-\mathrm{MeO}-$ phenethyl & $69,{ }^{\mathrm{a}} 73^{\mathrm{b}}$ \\
$\mathbf{g}$ & $\mathrm{Ph}$ & $\mathrm{Ph}$ & $26,{ }^{\mathrm{b}} 83,{ }^{\mathrm{c}} 80^{\mathrm{d}}$ \\
$\mathbf{h}$ & $\mathrm{Ph}$ & $3,4,5-(\mathrm{MeO})_{3} \mathrm{C}_{6} \mathrm{H}_{2}$ & $64,{ }^{\mathrm{b}} 80^{\mathrm{c}}$ \\
$\mathbf{i}$ & $4-t-\mathrm{BuC}_{6} \mathrm{H}_{4}$ & Cyclohexyl & $79^{\mathrm{a}}$ \\
$\mathbf{j}$ & $4-t-\mathrm{BuC}_{6} \mathrm{H}_{4}$ & $n-\mathrm{Heptyl}$ & $83,{ }^{\mathrm{a}} 82^{\mathrm{b}}$ \\
$\mathbf{k}$ & $4-t-\mathrm{BuC}_{6} \mathrm{H}_{4}$ & Phenethyl & $84,{ }^{\mathrm{a}} 82^{\mathrm{b}}$ \\
$\mathbf{l}$ & Octyl & Cyclohexyl & $69,{ }^{\mathrm{a}} 61^{\mathrm{b}}$ \\
$\mathbf{m}$ & Octyl & Phenethyl & $67,{ }^{\mathrm{a}} 70^{\mathrm{b}}$ \\
$\mathbf{n}$ & Octyl & $3,4,5-(\mathrm{MeO})_{3} \mathrm{C}_{6} \mathrm{H}_{2}$ & $58,{ }^{\mathrm{a}} 74^{\mathrm{b}}$ \\
\hline
\end{tabular}

${ }^{\text {a }}$ Method A: 2 equiv $\mathrm{K}_{2} \mathrm{CO}_{3}, \mathrm{DMF}, 160{ }^{\circ} \mathrm{C}$

b Method B: 2 equiv $\mathrm{K}_{2} \mathrm{CO}_{3}, \mathrm{Pd}\left(\mathrm{PPh}_{3}\right)_{4}(5 \mathrm{~mol} \%), \mathrm{DMF}, 110{ }^{\circ} \mathrm{C}$.

${ }^{c}$ Method C: 2 equiv $\mathrm{K}_{2} \mathrm{CO}_{3}, \mathrm{Pd}\left(\mathrm{PPh}_{3}\right)_{4}(5 \mathrm{~mol} \%)$, rac-BINAP $(5 \mathrm{~mol} \%)$, DMF, $110^{\circ} \mathrm{C}$.

${ }^{d}$ Method D: 2 equiv $\mathrm{K}_{2} \mathrm{CO}_{3}, \mathrm{Pd}\left(\mathrm{PPh}_{3}\right)_{4}(5 \mathrm{~mol} \%)$, DavePhos (7 mol \%), DMF, $110{ }^{\circ} \mathrm{C}$.

These results clearly show that the present approach could be applicable to various types of hetaryl ynones, providing a simple and efficient route to the synthesis of a wide range of the fused naphthyridine and quinoxaline derivatives, which are of interest as biologically active compounds. The structures of all the compounds were deduced from their spectral studies (IR, ${ }^{1} \mathrm{H},{ }^{13} \mathrm{C}$ NMR, and MS); most of the mass spectra displayed molecular ion peaks at the appropriate $m / z$ values. In addition, the structure of $\mathbf{6 i}$ was confirmed by X-ray single crystal analysis (Fig. 3). ${ }^{11}$

\section{Conclusion}

In conclusion, we have developed an efficient synthesis of benzo [b][1,8]naphthyridin-4(1H)-one and pyrido[2,3-b]quinoxalin-

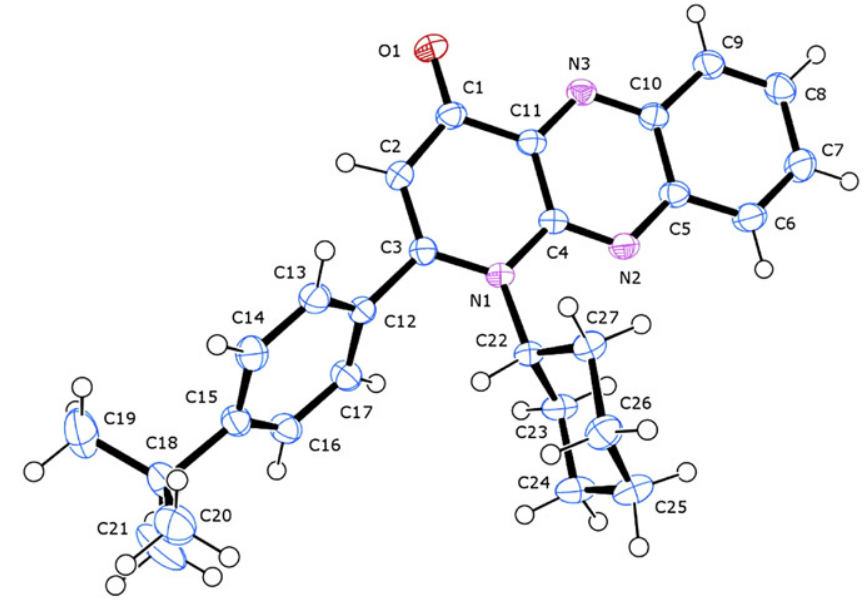

Fig. 3. Molecular structure of compound $\mathbf{6 i}$.

$4(1 \mathrm{H})$-one derivatives on the basis of readily available hetaryl alkynones. This palladium-catalyzed methodology is a valuable addition to heteroannulated pyridine synthesis and represents a general route to various nitrogen-containing heterocycles, which constitute an important structural subunit of a variety of biologically active compounds.

\section{Experimental}

\subsection{General}

NMR spectra were recorded on a Brucker AV 300 instruments. IR spectra were recorded on a Perkin Elmer FT IR 1600 spectrometer (ATR). Mass spectra were obtained on a Hewlett-Packard HPGC/MS $5890 / 5972$ instrument (EI, $70 \mathrm{eV}$ ) by GC inlet or on an MX-1321 instrument (EI, $70 \mathrm{eV}$ ) by direct inlet. Column chromatography was performed on silica gel (63-200 mesh, Merck) and silica gel Merck $60 \mathrm{~F}_{254}$ plates were used for TLC. All solvents were purified and dried by standard methods.

\subsection{General procedures for the synthesis of 2-phenylbenzo[b] $[1,8]$ naphthyridin-4(1H)-ones (3a-u)}

4.2.1. Method A (for $\mathbf{3 a}-\boldsymbol{l}$ ). A mixture of $\mathbf{2}(0.6 \mathrm{mmol})$, amine (0.7 mmol), $\mathrm{K}_{2} \mathrm{CO}_{3}(1.2 \mathrm{mmol})$, and $\mathrm{Pd}\left(\mathrm{PPh}_{3}\right)_{4}(0.05 \mathrm{mmol})$ was heated in $10 \mathrm{ml}$ DMF for $3 \mathrm{~h}$ at $170{ }^{\circ} \mathrm{C}$. The residue was purified by column chromatography on silica gel to afford the product.

4.2.2. Method B (for $\mathbf{3 m}-\boldsymbol{u}$ ). A mixture of $\mathbf{2}(0.6 \mathrm{mmol})$, amine (0.7 mmol), $\mathrm{Cs}_{2} \mathrm{CO}_{3}$ (235 mg, $0.72 \mathrm{mmol}$ ), $\mathrm{Pd}\left(\mathrm{PPh}_{3}\right)_{4}(5 \mathrm{~mol} \%)$, and toluene $(10 \mathrm{ml})$ was heated at $90^{\circ} \mathrm{C}$ for $6 \mathrm{~h}$. The residue was purified by column chromatography on silica gel to afford the product.

\subsection{General procedures for the synthesis of pyrido[2,3-b] quinoxalin-4(1H)-ones $(6 a-n)$}

4.3.1. Method A. Alkylamine (2.0 equiv), $\mathrm{K}_{2} \mathrm{CO}_{3}$ (2.0 equiv), and 1.0 equiv of the corresponding 1-(2-chloropyridin-3-yl)prop-2-yn1 -one 5 are heated at $160{ }^{\circ} \mathrm{C}$ in dry DMF under argon atmosphere in a pressure tube. After $16 \mathrm{~h}$, the solvent was removed in vacuo and the crude product was purified by column chromatography (eluent: $n$-heptane/ethylacetate).

4.3.2. Method B. Alkylamine (2.0 equiv), $\mathrm{K}_{2} \mathrm{CO}_{3}$ (2.0 equiv), $\mathrm{Pd}\left(\mathrm{PPh}_{3}\right)_{4}(5 \mathrm{~mol} \%)$, and 1.0 equiv of the corresponding 1-(2- 
chloropyridin-3-yl)prop-2-yn-1-one 5 are heated at $110{ }^{\circ} \mathrm{C}$ in dry DMF under argon atmosphere in a pressure tube. After approximately $16 \mathrm{~h}$ (controlled by TLC) the solvent was removed in vacuo and the crude product was purified by column chromatography (eluent: $n$-heptane-ethylacetate).

4.3.3. Method $C$. This method is similar to Method A, only the ligand rac-BINAP (5 mol \%) was loaded.

4.3.4. Method D. This method is similar to Method A, only the ligand DavePhos (7 mol \%) was loaded.

\section{Analytical data}

\subsection{1-(2-Chloroquinolin-3-yl)-3-phenylprop-2-yn-1-ol (1)}

Starting with 2-chloroquinoline-3-carbaldehyde (400 mg, $2.1 \mathrm{mmol}$ ), phenylacetylene (257 $\mathrm{mg}, 2.52 \mathrm{mmol}$ ), and $n$-BuLi ( $1.016 \mathrm{ml}, 2.52 \mathrm{mmol}), 1$ was isolated after column chromatography (silica gel, $n$-heptane/EtOAc $=5: 1$ ) as a white solid (600 mg, 98\%), mp 194-196 ${ }^{\circ} \mathrm{C} .{ }^{1} \mathrm{H}$ NMR (300 MHz, DMSO- $\left.d_{6}\right): \delta=5.95(\mathrm{~d}, 1 \mathrm{H}$, $\left.{ }^{3} J=5.6 \mathrm{~Hz}\right), 6.72\left(\mathrm{~d}, 1 \mathrm{H},{ }^{3} \mathrm{~J}=5.6 \mathrm{~Hz}\right), 7.43(\mathrm{~m}, 5 \mathrm{H}), 7.69(\mathrm{~m}, 1 \mathrm{H}), 7.90$ $(\mathrm{m}, 1 \mathrm{H}), 8.10\left(\mathrm{~d}, 1 \mathrm{H},{ }^{3} \mathrm{~J}=7.4 \mathrm{~Hz}\right), 8.20\left(\mathrm{~d}, 1 \mathrm{H},{ }^{3} \mathrm{~J}=6.9 \mathrm{~Hz}\right), 8.75(\mathrm{~s}, 1 \mathrm{H})$; ${ }^{13} \mathrm{C}$ NMR $\left(75 \mathrm{MHz}\right.$, DMSO- $\left.d_{6}\right): \delta=60.3,84.6,89.2,121.8,127.0,127.5$, 127.9, 128.3, 128.7, 128.8, 130.1, 130.9, 131.4, 133.5, 135.9, 136.5, 146.4, 148.3; IR (ATR, $\mathrm{cm}^{-1}$ ): $\nu=3233$ (w), 3065 (w), 2228 (w), 1591 (w), $1489(\mathrm{~m}), 1329(\mathrm{~m}), 1165(\mathrm{w}), 1068(\mathrm{~m}), 929(\mathrm{~m}), 857(\mathrm{w}), 779$ (m), 747 (s); GC-MS (EI, $70 \mathrm{eV}): m / z(\%)=293\left(\mathrm{M}^{+}\right), 258$ (100), 228 (24), 101 (10); HRMS (ESI-TOF): calcd for $\mathrm{C}_{18} \mathrm{H}_{12} \mathrm{NClO}[\mathrm{M}+\mathrm{H}]^{+}$ 294.06802, found 294.06773. Anal. Calcd for $\mathrm{C}_{18} \mathrm{H}_{12} \mathrm{ClNO}$ (293.747): C, 73.60; H, 4.12; N, 4.77. Found: C, 73.63; H, 4.15; N, 4.77.

\subsection{1-(2-Chloroquinolin-3-yl)-3-phenylprop-2-yn-1-one (2)}

Starting with 1-(2-chloroquinolin-3-yl)-3-phenylprop-2-yn-1ol 1 (400 mg, $1.36 \mathrm{mmol}$ ) and activated $\mathrm{MnO}_{2}$ (299 mg, $3.44 \mathrm{mmol}$ ), 2 was isolated after column chromatography (silica gel, $n$-heptane/EtOAc $=9: 1$ ) as a yellow solid (362 $\mathrm{mg}, 91 \%), \mathrm{mp}$ $101-103{ }^{\circ} \mathrm{C} .{ }^{1} \mathrm{H}$ NMR $\left(300 \mathrm{MHz}\right.$, DMSO- $\left.d_{6}\right): \delta=7.60(\mathrm{~m}, 3 \mathrm{H}), 7.82(\mathrm{~m}$, $3 \mathrm{H}), 8.03(\mathrm{~m}, 2 \mathrm{H}), 8.36\left(\mathrm{~d}, 1 \mathrm{H},{ }^{3} \mathrm{~J}=8.1 \mathrm{~Hz}\right), 9.30(\mathrm{~s}, 1 \mathrm{H}) ;{ }^{13} \mathrm{C} \mathrm{NMR}$ (75 MHz, DMSO- $\left.d_{6}\right): \delta=87.5,94.2,118.7,125.8,127.7,128.3,129.0$, 129.1, 129.2, 129.8, 131.8, 133.2, 133.3, 133.8, 144.1, 145.6, 147.7, 174.6; IR (ATR, cm $\left.{ }^{-1}\right): \nu=3062(\mathrm{w}), 2194(\mathrm{~m}), 1630(\mathrm{~s}), 1557(\mathrm{~m}), 1485(\mathrm{~m})$, 1392 (w), 1319 (m), 1212 (w), $1096(\mathrm{~s}), 1000$ (w), 978 (m), 805 (w), $775(\mathrm{~m}), 749(\mathrm{~s}), 597(\mathrm{~m})$; GC-MS (EI, $70 \mathrm{eV}): m / z(\%)=291\left(\mathrm{M}^{+}, 63\right)$, 263 (95), 228 (32), 129 (100), 101 (22), 75 (27); HRMS (ESI-TOF): calcd for $\mathrm{C}_{18} \mathrm{H}_{11} \mathrm{ClNO}[\mathrm{M}+\mathrm{H}]^{+}$292.05237, found 292.05241. Anal. Calcd for $\mathrm{C}_{18} \mathrm{H}_{10} \mathrm{ClNO}$ (291.731): C, 74.11; H, 3.46; N, 4.80. Found: C, 73.98; H, 3.56; N, 4.77.

\subsection{1-Cyclohexyl-2-phenylbenzo[b][1,8]naphthyridin-4(1H)- one (3a)}

Starting with 1-(2-chloroquinolin-3-yl)-3-phenylprop-2-yn-1one 2 (170 $\mathrm{mg}, 0.58 \mathrm{mmol})$, cyclohexylamine (70 $\mathrm{mg}, 0.7 \mathrm{mmol}$ ), potassium carbonate (162 $\mathrm{mg}, 1.17 \mathrm{mmol}), \mathrm{Pd}\left(\mathrm{PPh}_{3}\right)_{4}(5 \mathrm{~mol} \%)$, and DMF (10 ml) were refluxed at $170{ }^{\circ} \mathrm{C}$ for $3 \mathrm{~h}$, 3a was isolated after column chromatography (silica gel, $n$-heptane/EtOAc $=2: 1$ ) as a yellow solid (150 mg, 72\%), mp 280-282 ${ }^{\circ} \mathrm{C} .{ }^{1} \mathrm{H}$ NMR $(300 \mathrm{MHz}$, $\left.\mathrm{CDCl}_{3}\right): \delta=0.91-1.70(\mathrm{~m}, 8 \mathrm{H}), 3.15(\mathrm{~m}, 2 \mathrm{H}), 4.00(\mathrm{~m}, 1 \mathrm{H}), 6.08(\mathrm{~s}, 1 \mathrm{H})$, $7.39(\mathrm{~m}, 6 \mathrm{H}), 7.71(\mathrm{~m}, 1 \mathrm{H}), 7.94\left(\mathrm{dd}, 2 \mathrm{H},{ }^{3} \mathrm{~J}=8.3 \mathrm{~Hz},{ }^{4} \mathrm{~J}=1.5 \mathrm{~Hz}\right), 9.19$ (s, $1 \mathrm{H}) ;{ }^{13} \mathrm{C}$ NMR $\left(75 \mathrm{MHz}, \mathrm{CDCl}_{3}\right): \delta=25.3,26.5,26.6,30.5,63.8,111.4$, $121.5,125.1,125.6,127.4,127.5,128.1,128.8,129.2,129.4,131.9,137.1$, 137.2, 148.3, 150.1, 158.3, 178.6; IR (ATR, $\left.\mathrm{cm}^{-1}\right): \nu=3046(\mathrm{w}), 2940$ (m), $1616(\mathrm{~m}), 1578(\mathrm{~s}), 1420(\mathrm{~m}), 1164(\mathrm{w}), 1116(\mathrm{~m}), 1041(\mathrm{~m}), 893$ (w), $976(\mathrm{~m}), 844(\mathrm{~s}), 796(\mathrm{~m}), 758(\mathrm{~s}), 617(\mathrm{~m}), 537$ (m); GC-MS (EI,
$70 \mathrm{eV}): m / z(\%)=354\left(\mathrm{M}^{+}, 6\right), 273$ (23), 272 (100), 244 (31); HRMS (ESI-TOF): calcd for $\mathrm{C}_{24} \mathrm{H}_{23} \mathrm{~N}_{2} \mathrm{O} \quad[\mathrm{M}+\mathrm{H}]^{+}$355.18049, found 355.18054. Anal. Calcd for $\mathrm{C}_{24} \mathrm{H}_{22} \mathrm{~N}_{2} \mathrm{O}$ (354.444): C, 81.33; H, 6.26; N, 7.90. Found: C, 80.98; H, 6.46; N, 7.75.

\subsection{1-Benzyl-2-phenylbenzo[b][1,8]naphthyridin- $4(1 H)$-one (3b)}

Starting with 1-(2-chloroquinolin-3-yl)-3-phenylprop-2-yn-1one 2 (170 $\mathrm{mg}, 0.58 \mathrm{mmol}$ ), benzylamine ( $75 \mathrm{mg}, 0.7 \mathrm{mmol})$, potassium carbonate (162 mg, $1.17 \mathrm{mmol}), \mathrm{Pd}\left(\mathrm{PPh}_{3}\right)_{4}(5 \mathrm{~mol} \%)$, and DMF (10 ml) were refluxed at $170{ }^{\circ} \mathrm{C}$ for $3 \mathrm{~h}, \mathbf{3 b}$ was isolated after column chromatography (silica gel, $n$-heptane/EtOAc $=2: 1$ ) as a yellow solid (180 mg, 85\%), $\mathrm{mp} 238^{\circ} \mathrm{C} .{ }^{1} \mathrm{H} \mathrm{NMR}\left(\mathrm{CDCl}_{3}, 300 \mathrm{MHz}\right)$ : $\delta=5.71(\mathrm{~s}, 2 \mathrm{H}), 6.21(\mathrm{~s}, 1 \mathrm{H}), 6.78-6.81(\mathrm{~m}, 2 \mathrm{H}), 7.06-7.08(\mathrm{~m}, 3 \mathrm{H})$, 7.18-7.22 (m, 2H), 7.28-7.49 (m, 4H), 7.69-7.74 (m, 1H), $7.91(\mathrm{~d}, 1 \mathrm{H}$, $\left.{ }^{3} \mathrm{~J}=8.1 \mathrm{~Hz}\right), 7.98\left(\mathrm{~d}, 1 \mathrm{H},{ }^{3} \mathrm{~J}=8.1 \mathrm{~Hz}\right), 9.26(\mathrm{~s}, 1 \mathrm{H}) ;{ }^{13} \mathrm{C}$ NMR $(75 \mathrm{MHz}$, $\left.\mathrm{CDCl}_{3}\right): \delta=49.4,111.6,120.6,125.5,125.7,126.7,127.1,128.2,128.3$, $128.4,128.5,129.3,129.6,132.2,135.6,137.7,137.9,149.1,149.6$, 157.4, 178.8; IR (ATR, $\mathrm{cm}^{-1}$ ): $\nu=3048$ (w), $1616(\mathrm{~s}), 1552(\mathrm{~m}), 1494$ (w), 1328 (m), $1184(\mathrm{w}), 1145$ (m), $1072(\mathrm{w}), 1023(\mathrm{~m}), 928(\mathrm{w}), 831$ (s), 790 (m), 755 (s), 609 (m); GC-MS (EI, $70 \mathrm{eV}): \mathrm{m} / \mathrm{z}(\%)=362\left(\mathrm{M}^{+}\right.$, 63), 271 (100); HRMS (ESI-TOF): calcd for $\mathrm{C}_{25} \mathrm{H}_{18} \mathrm{~N}_{2} \mathrm{O}[\mathrm{M}+\mathrm{H}]^{+}$ 363.42322; found 363.42323. Anal. Calcd for $\mathrm{C}_{25} \mathrm{H}_{18} \mathrm{~N}_{2} \mathrm{O}$ (362.42322): C, 82.85; H, 5.01; N, 7.73. Found: C, 82.98; H, 5.21; N, 7.86 .

\subsection{1-(4-Methoxybenzyl)-2-phenylbenzo[b][1,8]naphthyr- idin-4(1H)-one (3c)}

Starting with 1-(2-chloroquinolin-3-yl)-3-phenylprop-2-yn-1one 2 (170 mg, $0.58 \mathrm{mmol}$ ), p-methoxybenzylamine (96 mg, $0.7 \mathrm{mmol})$, potassium carbonate (162 mg, $1.17 \mathrm{mmol}), \mathrm{Pd}\left(\mathrm{PPh}_{3}\right)_{4}$ ( $5 \mathrm{~mol} \%)$, and DMF $\left(10 \mathrm{ml}\right.$ ) were refluxed at $170{ }^{\circ} \mathrm{C}$ for $3 \mathrm{~h}, 3 \mathrm{c}$ was isolated after column chromatography (silica gel, $n$-heptane/ EtOAc $=3: 1)$ as a yellow solid $(146 \mathrm{mg}, 64 \%), \mathrm{mp} 174-176{ }^{\circ} \mathrm{C} .{ }^{1} \mathrm{H}$ NMR (300 MHz, $\left.\mathrm{CDCl}_{3}\right): \delta=3.64(\mathrm{~s}, 3 \mathrm{H}), 5.65(\mathrm{~s}, 2 \mathrm{H}), 6.59(\mathrm{~d}, 2 \mathrm{H}$, $\left.{ }^{3} \mathrm{~J}=9.7 \mathrm{~Hz}\right), 6.74\left(\mathrm{~d}, 2 \mathrm{H},{ }^{3} \mathrm{~J}=9.6 \mathrm{~Hz}\right), 7.21(\mathrm{~m}, 3 \mathrm{H}), 7.41(\mathrm{~m}, 4 \mathrm{H}), 7.73$ $(\mathrm{m}, 1 \mathrm{H}), 7.96$ (dd, $\left.2 \mathrm{H},{ }^{3} \mathrm{~J}=9.7 \mathrm{~Hz},{ }^{4} \mathrm{~J}=3.1 \mathrm{~Hz}\right), 9.25(\mathrm{~s}, 1 \mathrm{H}) ;{ }^{13} \mathrm{C}$ NMR $\left(75 \mathrm{MHz}, \mathrm{CDCl}_{3}\right): \delta=48.8,55.2,111.7,113.6,120.7,125.5,125.7,128.2$, 128.3, 128.4, 128.5, 129.3, 129.5, 129.9, 132.2, 135.7, 137.5, 137.6, 149.6, 157.4, 158.7, 178.8; IR (ATR, $\mathrm{cm}^{-1}$ ): $\nu=2958$ (w), 1580 (s), 1511 (m), $1478(\mathrm{~s}), 1282(\mathrm{~m}), 1245(\mathrm{~s}), 1145(\mathrm{~m}), 845(\mathrm{~m}), 828(\mathrm{~s}), 787(\mathrm{~m})$, 568 (m); GC-MS (EI, $70 \mathrm{eV}): \mathrm{m} / z(\%)=391\left(\mathrm{M}^{+}, 63\right), 263$ (95), 228 (32), 129 (100), 101 (22), 75 (27); HRMS (ESI-TOF): calcd for $\mathrm{C}_{26} \mathrm{H}_{21} \mathrm{~N}_{2} \mathrm{O}_{2}[\mathrm{M}+\mathrm{H}]^{+}$393.15975, found 393.16055. Anal. Calcd for $\mathrm{C}_{26} \mathrm{H}_{20} \mathrm{~N}_{2} \mathrm{O}_{2}$ (392.449): C, 79.57; H, 5.14; N, 7.14. Found: C, 79.69; H, 5.26; N, 7.04 .

\subsection{1-Phenethyl-2-phenylbenzo[b][1,8]naphthyridin-4(1H)- one (3d)}

Starting with 1-(2-chloroquinolin-3-yl)-3-phenylprop-2-yn-1one 2 (170 mg, $0.58 \mathrm{mmol}$ ), 2-phenylethanamine $(85 \mathrm{mg}$, $0.7 \mathrm{mmol})$, potassium carbonate (162 $\mathrm{mg}, 1.17 \mathrm{mmol}), \mathrm{Pd}\left(\mathrm{PPh}_{3}\right)_{4}$ ( $5 \mathrm{~mol} \%$ ), and DMF (10 ml) were refluxed at $170{ }^{\circ} \mathrm{C}$ for $3 \mathrm{~h}$, 3d was isolated after column chromatography (silica gel, $n$-heptane/ EtOAc $=3: 1)$ as a yellow solid (97 mg, 46\%), mp $232{ }^{\circ} \mathrm{C} .{ }^{1} \mathrm{H}$ NMR $\left(300 \mathrm{MHz} \mathrm{CDCl}_{3}\right): \delta=2.81\left(\mathrm{t}, 2 \mathrm{H},{ }^{3} \mathrm{~J}=8.1 \mathrm{~Hz}\right), 4.42\left(\mathrm{t}, 2 \mathrm{H},{ }^{3} \mathrm{~J}=8.1 \mathrm{~Hz}\right)$, $6.01(\mathrm{~s}, 1 \mathrm{H}), 6.71\left(\mathrm{~d}, 2 \mathrm{H},{ }^{3} \mathrm{~J}=6.7 \mathrm{~Hz}\right), 6.98-7.04(\mathrm{~m}, 3 \mathrm{H}), 7.09-7.12$ $(\mathrm{m}, 2 \mathrm{H}), 7.28-7.39(\mathrm{~m}, 4 \mathrm{H}), 7.63-7.68(\mathrm{~m}, 1 \mathrm{H}), 7.90\left(\mathrm{~d}, 2 \mathrm{H},{ }^{3} \mathrm{~J}=9.3\right.$, $8.1 \mathrm{~Hz}), 9.13(\mathrm{~s}, 1 \mathrm{H}) ;{ }^{13} \mathrm{C} \mathrm{NMR}\left(75 \mathrm{MHz}, \mathrm{CDCl}_{3}\right): \delta=35.4,48.4,110.9$, $125.5,125.8,126.6,128.1,128.1,128.3,128.3,128.6,128.6,128.7$, 128.7, 128.8, 129.4, 129.6, 132.4, 135.6, 137.7, 138.2, 148.2, 148.5, 149.1, 149.3, 177.4; IR (ATR, $\left.\mathrm{cm}^{-1}\right): \nu=3044(\mathrm{w}), 1582(\mathrm{~s}), 1552(\mathrm{~m})$, $1514(\mathrm{w}), 1477$ (s), 1445 (m), 1329 (m), 1146 (m), 976 (m), $829(\mathrm{~s})$, 
573 (s); GC-MS (EI, $70 \mathrm{eV}): m / z(\%)=377(17), 376\left(\mathrm{M}^{+}, 56\right), 375$ (100), 180 (28), 105 (28); HRMS (ESI-TOF): calcd for $\mathrm{C}_{26} \mathrm{H}_{21} \mathrm{~N}_{2} \mathrm{O}$ $[\mathrm{M}+\mathrm{H}]^{+}$377.16484, found 377.16543. Anal. Calcd for $\mathrm{C}_{26} \mathrm{H}_{20} \mathrm{~N}_{2} \mathrm{O}$ (376.450): C, 82.95; H, 5.35; N, 7.44. Found: C, 82.73; H, 5.26; N, 7.40 .

\subsection{1-Heptyl-2-phenylbenzo[b][1,8]naphthyridin-4(1H)-one (3e)}

Starting with 1-(2-chloroquinolin-3-yl)-3-phenylprop-2-yn-1one 2 (170 $\mathrm{mg}, 0.58 \mathrm{mmol}), n$-heptylamine $(81 \mathrm{mg}, 0.7 \mathrm{mmol})$, potassium carbonate (162 mg, $1.17 \mathrm{mmol}), \mathrm{Pd}\left(\mathrm{PPh}_{3}\right)_{4}(5 \mathrm{~mol} \%)$, and DMF $(10 \mathrm{ml})$ were refluxed at $170{ }^{\circ} \mathrm{C}$ for $3 \mathrm{~h}$, 3e was isolated after column chromatography (silica gel, $n$-heptane/EtOAc $=2: 1$ ) as a yellow solid (140 mg, 65\%), $\left.\mathrm{mp} 315^{\circ} \mathrm{C} .{ }^{1} \mathrm{H} \mathrm{NMR} \mathrm{(300} \mathrm{MHz,} \mathrm{CDCl}_{3}\right)$ : $\delta=0.76\left(\mathrm{t}, 3 \mathrm{H},{ }^{3} \mathrm{~J}=7.5 \mathrm{~Hz}\right), 1.13(\mathrm{~m}, 8 \mathrm{H}), 1.62\left(\mathrm{t}, 2 \mathrm{H},{ }^{3} \mathrm{~J}=7.5 \mathrm{~Hz}\right), 4.34(\mathrm{t}$, $\left.2 \mathrm{H},{ }^{3} \mathrm{~J}=8.1 \mathrm{~Hz}\right), 6.14(\mathrm{~s}, 1 \mathrm{H}), 7.44(\mathrm{~m}, 6 \mathrm{H}), 7.75(\mathrm{~m}, 1 \mathrm{H}), 7.98(\mathrm{~d}, 2 \mathrm{H}$, $\left.{ }^{3} \mathrm{~J}=8.5 \mathrm{~Hz}\right), 9.24(\mathrm{~s}, 1 \mathrm{H}) ;{ }^{13} \mathrm{C} \mathrm{NMR}\left(\mathrm{CDCl}_{3}, 75 \mathrm{MHz}\right): \delta=14.1,22.5$, 26.5, 28.5, 28.9, 31.3, 46.7, 111.0, 120.7, 125.3, 125.5, 127.8, 128.1, $128.1,128.5,128.6,129.2,129.4,132.0,135.9,137.3,148.9,149.1,157.1$, 178.5; IR (ATR, $\mathrm{cm}^{-1}$ ): $\nu=3048$ (w), 2918 (m), 1632 (s), 1411 (m), $1332(\mathrm{~m}), 1126(\mathrm{w}), 1074(\mathrm{w}), 976(\mathrm{~m}), 774(\mathrm{~m}), 753(\mathrm{~s}), 719(\mathrm{~m}), 611$ (m); GC-MS (EI, $70 \mathrm{eV}): m / z(\%)=370\left(\mathrm{M}^{+}, 27\right), 313$ (12), 285 (40), 272 (100), 244 (25), 128 (11); HRMS (ESI-TOF): calcd for $\mathrm{C}_{25} \mathrm{H}_{27} \mathrm{~N}_{2} \mathrm{O}$ $[\mathrm{M}+\mathrm{H}]^{+}$371.21179, found 371.21180. Anal. Calcd for $\mathrm{C}_{25} \mathrm{H}_{26} \mathrm{~N}_{2} \mathrm{O}$ (370.487): C, 81.05; H, 7.07; N, 7.56. Found: C, 80.98; H, 7.08, N, 7.49.

\subsection{1-Cyclopropyl-2-phenylbenzo[b][1,8]naphthyridin-4- (1H)-one (3f)}

Starting with 1-(2-chloroquinolin-3-yl)-3-phenylprop-2-yn-1one 2 (170 mg, $0.58 \mathrm{mmol}$ ), cyclopropylamine ( $40 \mathrm{mg}, 0.7 \mathrm{mmol})$, potassium carbonate $(162 \mathrm{mg}, 1.17 \mathrm{mmol}), \mathrm{Pd}\left(\mathrm{PPh}_{3}\right)_{4}(5 \mathrm{~mol} \%)$, and DMF (10 ml) were refluxed at $170{ }^{\circ} \mathrm{C}$ for $3 \mathrm{~h}$, 3f was isolated after column chromatography (silica gel, $n$-heptane/EtOAc $=2: 1$ ) as a yellow solid (61 mg, 57\%), mp $193{ }^{\circ} \mathrm{C} .{ }^{1} \mathrm{H}$ NMR (250 MHz, $\left.\mathrm{CDCl}_{3}\right)$ : $\delta=0.94(\mathrm{~m}, 2 \mathrm{H}), 1.46(\mathrm{~m}, 2 \mathrm{H}), 3.45(\mathrm{~m}, 1 \mathrm{H}), 6.24(\mathrm{~s}, 1 \mathrm{H}), 7.50(\mathrm{~m}, 6 \mathrm{H})$, $7.78(\mathrm{~m}, 1 \mathrm{H}), 7.99\left(\mathrm{~d}, 1 \mathrm{H},{ }^{3} \mathrm{~J}=9.7 \mathrm{~Hz}\right), 8.10\left(\mathrm{~d}, 1 \mathrm{H},{ }^{3} \mathrm{~J}=9.7 \mathrm{~Hz}\right), 9.20(\mathrm{~s}$, $1 \mathrm{H}) ;{ }^{13} \mathrm{C} \mathrm{NMR}\left(\mathrm{CDCl}_{3}, 75 \mathrm{MHz}\right): \delta=12.7,32.2,111.6,120.8,125.4$, 125.6, 128.2, 128.4, 128.5, 129.3, 129.4, 132.1, 136.8, 137.1, 148.9, 151.7, 158.2, 179.3; IR (KBr, cm $\left.{ }^{-1}\right): \nu=3060$ (w), 1633 (s), 1616 (m), 1350 (w), $1263(\mathrm{~m}), 1143(\mathrm{w}), 1031(\mathrm{~m}), 931(\mathrm{w}), 885(\mathrm{w}), 791(\mathrm{~m}), 773(\mathrm{~s})$, $744(\mathrm{~s}), 605(\mathrm{~m})$; GC-MS (EI, $70 \mathrm{eV}): m / z(\%)=312\left(\mathrm{M}^{+}, 25\right), 311$ (100), 283 (21); HRMS (ESI-TOF): calcd for $\mathrm{C}_{21} \mathrm{H}_{17} \mathrm{~N}_{2} \mathrm{O}[\mathrm{M}+\mathrm{H}]^{+}$ 313.13354, found 313.13355. Anal. Calcd for $\mathrm{C}_{21} \mathrm{H}_{16} \mathrm{~N}_{2} \mathrm{O}$ (312.126): $\mathrm{C}$, 80.75; H, 5.16; N, 8.97. Found: C, 80.98; H, 5.06; N, 8.97.

\subsection{2-Phenylbenzo[b][1,8]naphthyridin-4(1H)-one (3g)}

Starting with 1-(2-chloroquinolin-3-yl)-3-phenylprop-2-yn-1one 2 (170 $\mathrm{mg}, 0.58 \mathrm{mmol}$ ), allylamine ( $40 \mathrm{mg}, 0.7 \mathrm{mmol})$, potassium carbonate (162 mg, $1.17 \mathrm{mmol}), \mathrm{Pd}\left(\mathrm{PPh}_{3}\right)_{4}(5 \mathrm{~mol} \%)$, and DMF $\left(10 \mathrm{ml}\right.$ ) were refluxed at $170^{\circ} \mathrm{C}$ for $3 \mathrm{~h}, 3 \mathrm{~g}$ was isolated after column chromatography ( silica gel, $n$-heptane/EtOAc $=2: 1$ ) as a yellow solid (141 mg, 90\%), mp 286-288 ${ }^{\circ} \mathrm{C} .{ }^{1} \mathrm{H}$ NMR (250 MHz, $\left.\mathrm{CDCl}_{3}\right): \delta=6.37$ $(\mathrm{s}, 1 \mathrm{H}), 7.58(\mathrm{~m}, 5 \mathrm{H}), 7.90(\mathrm{~m}, 3 \mathrm{H}), 7.99\left(\mathrm{~d}, 1 \mathrm{H},{ }^{3} \mathrm{~J}=8.0 \mathrm{~Hz}\right), 8.23(\mathrm{~d}, 1 \mathrm{H}$, $\left.{ }^{3} \mathrm{~J}=8.6 \mathrm{~Hz}\right), 9.20(\mathrm{~s}, 1 \mathrm{H}), 12.33(\mathrm{~s}, 1 \mathrm{H}, \mathrm{NH}) ;{ }^{13} \mathrm{C} \mathrm{NMR}\left(\mathrm{CDCl}_{3}, 75 \mathrm{MHz}\right)$ : $\delta=106.2,119.3,125.2,126.7,127.7,127.7,128.7,128.8,129.8,130.8$, 132.5, 133.6, 136.6, 148.7, 149.0, 150.2, 153.1, 178.2; IR (ATR, $\left.\mathrm{cm}^{-1}\right)$ : $\nu=1650(\mathrm{w}), 1540(\mathrm{~m}), 1454(\mathrm{~m}), 1227(\mathrm{w}), 1266(\mathrm{~m}), 1154(\mathrm{w}), 958$ (w), $855(\mathrm{~m}), 791(\mathrm{~m}), 740$ (s), 690 (s), 613 (m), 547 (s); GC-MS (EI, $70 \mathrm{eV}): m / z(\%)=273(18), 272\left(\mathrm{M}^{+}, 100\right), 271(13), 245$ (18), 244 (78), 243 (14), 242 (11), 122 (11); HRMS (ESI-TOF): calcd for $\mathrm{C}_{18} \mathrm{H}_{13} \mathrm{~N}_{2} \mathrm{O}[\mathrm{M}+\mathrm{H}]^{+}$273.10224, found 273.10223. Anal. Calcd for $\mathrm{C}_{18} \mathrm{H}_{12} \mathrm{~N}_{2} \mathrm{O}$ (272.095): C, 79.39; H, 4.44; N, 10.29. Found: C, 79.19; H, $4.46 ; \mathrm{N}, 10.26$.

\subsection{1-(3-Morpholinopropyl)-2-phenylbenzo[b][1,8]naph- thyridin-4(1H)-one (3h)}

Starting with 1-(2-chloroquinolin-3-yl)-3-phenylprop-2-yn-1one 2 (170 $\mathrm{mg}, 0.58 \mathrm{mmol}), 3$-(4-morpholino)propylamine (101 $\mathrm{mg}, 0.7 \mathrm{mmol})$, potassium carbonate $(162 \mathrm{mg}, 1.17 \mathrm{mmol}$ ), $\mathrm{Pd}\left(\mathrm{PPh}_{3}\right)_{4}(5 \mathrm{~mol} \%)$, and $\mathrm{DMF}(10 \mathrm{ml})$ were reflux at $170{ }^{\circ} \mathrm{C}$ for $3 \mathrm{~h}$, 3h was isolated after column chromatography (silica gel, $n$-heptane/EtOAc $=2: 1)$ as a solid $(200 \mathrm{mg}, 87 \%), \mathrm{mp} 188-189{ }^{\circ} \mathrm{C} .{ }^{1} \mathrm{H}$ NMR $\left(\mathrm{CDCl}_{3}, 300 \mathrm{MHz}\right): \delta=1.76(\mathrm{~m}, 2 \mathrm{H}), 2.17(\mathrm{~m}, 6 \mathrm{H}), 3.49(\mathrm{t}, 4 \mathrm{H}$, $\left.{ }^{3} \mathrm{~J}=10.2 \mathrm{~Hz}\right), 4.43\left(\mathrm{dd}, 2 \mathrm{H},{ }^{3} \mathrm{~J}=8.1 \mathrm{~Hz},{ }^{4} \mathrm{~J}=2.7 \mathrm{~Hz}\right), 6.11(\mathrm{~s}, 1 \mathrm{H}), 7.43(\mathrm{~m}$, $6 \mathrm{H}), 7.74(\mathrm{~m}, 1 \mathrm{H}), 7.94\left(\mathrm{dd}, 2 \mathrm{H},{ }^{3} \mathrm{~J}=12.5 \mathrm{~Hz},{ }^{4} \mathrm{~J}=3.2 \mathrm{~Hz}\right), 9.22(\mathrm{~s}, 1 \mathrm{H})$; ${ }^{13} \mathrm{C}$ NMR $\left(75 \mathrm{MHz} \mathrm{CDCl}_{3}\right): \delta=25.8,45.1,53.4,53.4,55.9,66.8,66.9$, $111.2,120.9,125.4,125.6,128.1,128.2,128.2,128.7,128.7,129.4$, 129.5, 132.2, 135.8, 137.6, 149.1, 149.3, 157.0, 178.6; IR (ATR, $\left.\mathrm{cm}^{-1}\right)$ : $\nu=2914$ (w), 2814 (w), 1633 (s), 1551 (m), 1474 (m), 1353 (w), 1264 (m), 1114 (s), $1036(\mathrm{~m}), 995$ (w), 937 (m), 835 (s); GC-MS (EI, $70 \mathrm{eV}): m / z(\%)=398\left(\mathrm{M}^{+}, 73\right), 313$ (20), 299 (23), 285 (100), 273 (66), 244 (13), 128 (18), 100 (26); HRMS (ESI-TOF): calcd for $\mathrm{C}_{25} \mathrm{H}_{26} \mathrm{~N}_{3} \mathrm{O}_{2}[\mathrm{M}+\mathrm{H}]^{+}$400.20195, found 400.20292. Anal. Calcd for $\mathrm{C}_{25} \mathrm{H}_{25} \mathrm{~N}_{3} \mathrm{O}_{2}$ (399.195): C, 75.16; $\mathrm{H}, 6.31 ; \mathrm{N}, 10.52$. Found: C, 74.98; $\mathrm{H}$, $6.56 ; \mathrm{N}, 10.54$.

\subsection{1-(2-Hydroxyethyl)-2-phenylbenzo[b][1,8]naphthyridin- $4(1 H)$-one (3i)}

Starting with 1-(2-chloroquinolin-3-yl)-3-phenylprop-2-yn-1one 2 (170 $\mathrm{mg}, 0.58 \mathrm{mmol}$ ), ethanolamine (32 $\mathrm{mg}, 0.7 \mathrm{mmol}$ ), potassium carbonate (162 mg, $1.17 \mathrm{mmol}), \mathrm{Pd}\left(\mathrm{PPh}_{3}\right)_{4}(5 \mathrm{~mol} \%)$, and DMF $(10 \mathrm{ml})$ were refluxed at $170^{\circ} \mathrm{C}$ for $3 \mathrm{~h}, 3 \mathbf{i}$ was isolated after column chromatography (silica gel, $n$-heptane/EtOAc $=2: 1$ ) as a yellow solid (150 mg, 72\%), $\mathrm{mp} 204{ }^{\circ} \mathrm{C} .{ }^{1} \mathrm{H} \mathrm{NMR}\left(\mathrm{CDCl}_{3}, 300 \mathrm{MHz}\right)$ : $\delta=4.01\left(\mathrm{dd}, 2 \mathrm{H},{ }^{3} \mathrm{~J}=9.3 \mathrm{~Hz},{ }^{4} \mathrm{~J}=3.1 \mathrm{~Hz}\right), 4.50\left(\mathrm{dd}, 2 \mathrm{H},{ }^{3} \mathrm{~J}=8.4 \mathrm{~Hz}\right.$, $\left.{ }^{4} \mathrm{~J}=2.9 \mathrm{~Hz}\right), 4.86\left(\mathrm{t}, 1 \mathrm{H},{ }^{3} \mathrm{~J}=12.1 \mathrm{~Hz}\right), 6.18(\mathrm{~s}, 1 \mathrm{H}), 7.50(\mathrm{~m}, 6 \mathrm{H}), 7.82$ $(\mathrm{m}, 1 \mathrm{H}), 7.92\left(\mathrm{~d}, 1 \mathrm{H},{ }^{3} \mathrm{~J}=7.8 \mathrm{~Hz}\right), 8.03\left(\mathrm{~d}, 1 \mathrm{H},{ }^{3} \mathrm{~J}=7.9 \mathrm{~Hz}\right), 9.10(\mathrm{~s}, 1 \mathrm{H})$; ${ }^{13} \mathrm{C} \mathrm{NMR}\left(\mathrm{CDCl}_{3}, 75 \mathrm{MHz}\right): \delta=50.5,62.5,111.6,120.7,125.4,125.9$, $127.5,128.5,128.8,129.3,129.7,132.7,135.8,138.1,148.5,150.2$, 157.8, 178.4; IR (ATR, $\mathrm{cm}^{-1}$ ): $\nu=3206$ (w), 1614 (s), 1480 (s), 1338 (m), $1293(\mathrm{~m}), 1176(\mathrm{w}), 1156(\mathrm{~m}), 1066(\mathrm{~m}), 959(\mathrm{w}), 825(\mathrm{~s}), 612$ (m), 559 (m); GC-MS (EI, $70 \mathrm{eV}): m / z(\%)=316\left(\mathrm{M}^{+}, 12\right), 297(19)$, 285 (67), 284 (16), 273 (20), 272 (100), 255 (11), 128 (22); HRMS (ESI-TOF): calcd for $\mathrm{C}_{20} \mathrm{H}_{17} \mathrm{~N}_{2} \mathrm{O}_{2} \quad[\mathrm{M}+\mathrm{H}]^{+}$317.12845, found 317.12854. Anal. Calcd for $\mathrm{C}_{20} \mathrm{H}_{16} \mathrm{~N}_{2} \mathrm{O}_{2}$ (316.353): C, 75.93; $\mathrm{H}, 5.10$; N, 8.86. Found: C, 75.90; H, 5.08; N, 8.86.

\subsection{1-(3-Methoxybenzyl)-2-phenylbenzo $[b][1,8]$ naphthyr- idin-4(1H)-one (3j)}

Starting with 1-(2-chloroquinolin-3-yl)-3-phenylprop-2-yn-1one 2 (170 mg, $0.58 \mathrm{mmol}$ ), 3-methoxybenzylamine (96 mg, $0.7 \mathrm{mmol})$, potassium carbonate (162 $\mathrm{mg}, 1.17 \mathrm{mmol}), \mathrm{Pd}\left(\mathrm{PPh}_{3}\right)_{4}$ (5 mol \%), and DMF (10 ml) were refluxed at $170{ }^{\circ} \mathrm{C}$ for $3 \mathrm{~h}, \mathbf{3 j}$ was isolated after column chromatography (silica gel, $n$-heptane/ EtOAc $=2: 1)$ as a solid $(120 \mathrm{mg}, 52 \%), \mathrm{mp} 114-116{ }^{\circ} \mathrm{C} .{ }^{1} \mathrm{H}$ NMR (300 MHz, $\left.\mathrm{CDCl}_{3}\right): \delta=3.67(\mathrm{~s}, 3 \mathrm{H}), 6.29(\mathrm{~s}, 1 \mathrm{H}), 6.48-6.51(\mathrm{~m}, 2 \mathrm{H})$, $6.72\left(\mathrm{~d}, 1 \mathrm{H},{ }^{3} \mathrm{~J}=5.3 \mathrm{~Hz}\right), 7.07\left(\mathrm{t}, 1 \mathrm{H},{ }^{3} \mathrm{~J}=8.1 \mathrm{~Hz}\right), 7.28-7.59(\mathrm{~m}, 7 \mathrm{H})$, $7.79-7.85(\mathrm{~m}, 1 \mathrm{H}), 8.02\left(\mathrm{~d}, 1 \mathrm{H},{ }^{3} \mathrm{~J}=8.1 \mathrm{~Hz}\right), 8.09\left(\mathrm{~d}, 1 \mathrm{H},{ }^{3} \mathrm{~J}=8.1 \mathrm{~Hz}\right)$, $9.36(\mathrm{~s}, 1 \mathrm{H}) ;{ }^{13} \mathrm{C}$ NMR $\left(\mathrm{CDCl}_{3}, 75 \mathrm{MHz}\right): \delta=49.3,55.1,111.7,112.3$, 112.7, 119.1, 120.6, 125.5, 125.7, 128.2, 128.3, 128.5, 129.3, 129.3, 129.6, 132.3, 135.7, 137.7, 139.6, 149.2, 149.6, 157.5, 159.6, 178.9; IR (ATR, $\left.\mathrm{cm}^{-1}\right): \nu=2921(\mathrm{w}), 1660(\mathrm{~m}), 1631(\mathrm{~m}), 1582(\mathrm{~s}), 1486(\mathrm{w})$, $1349(\mathrm{~m}), 1274(\mathrm{~m}), 1158(\mathrm{~m}), 1092(\mathrm{~m}), 976(\mathrm{~m}), 847(\mathrm{~m}), 698(\mathrm{~s})$; GC-MS (EI, $70 \mathrm{eV}): m / z(\%)=393(11), 392\left(\mathrm{M}^{+}, 54\right), 391$ (100), 283 (10), 121 (17); HRMS (EI): calcd for $\mathrm{C}_{26} \mathrm{H}_{19} \mathrm{~N}_{2} \mathrm{O}_{2}$ [M] 391.14410 , found 391.14442. Anal. Calcd for $\mathrm{C}_{20} \mathrm{H}_{19} \mathrm{~N}_{2} \mathrm{O}_{2}$ (392.449): $\mathrm{C}, 74.11 ; \mathrm{H}$, 3.46; N, 4.8. Found: C, 73.98; H, 3.56; N, 4.77. 
5.13. 1-(2-Chlorobenzyl)-2-phenylbenzo[b][1,8]naphthyridin4(1H)-one (3k)

Starting with 1-(2-chloroquinolin-3-yl)-3-phenylprop-2-yn-1one 2 (170 mg, $0.58 \mathrm{mmol}$ ), 2-chlorobenzylamine (99 $\mathrm{mg}$, $0.7 \mathrm{mmol}$ ), potassium carbonate (162 $\mathrm{mg}, 1.17 \mathrm{mmol}), \mathrm{Pd}\left(\mathrm{PPh}_{3}\right)_{4}$ (5 $\mathrm{mol} \%)$, and DMF (10 ml) were refluxed at $170{ }^{\circ} \mathrm{C}$ for $3 \mathrm{~h}, \mathbf{3 k}$ was isolated after column chromatography (silica gel, $n$-heptane/ EtOAc $=2: 1)$ as a yellow solid $(200 \mathrm{mg}, 86 \%), \mathrm{mp} 238{ }^{\circ} \mathrm{C} .{ }^{1} \mathrm{H}$ NMR $\left(\mathrm{CDCl}_{3}, 300 \mathrm{MHz}\right): \delta=5.81(\mathrm{~s}, 2 \mathrm{H}), 6.33(\mathrm{~s}, 1 \mathrm{H}), 6.76(\mathrm{~d}, 1 \mathrm{H}$, $\left.{ }^{3} \mathrm{~J}=8.6 \mathrm{~Hz}\right), 7.17(\mathrm{~m}, 1 \mathrm{H}), 7.21(\mathrm{~m}, 3 \mathrm{H}), 7.47(\mathrm{~m}, 4 \mathrm{H}), 7.79(\mathrm{~m}, 1 \mathrm{H}), 7.95$ (d, $\left.1 \mathrm{H},{ }^{3} \mathrm{~J}=8.7 \mathrm{~Hz}\right), 8.07$ (d, $\left.1 \mathrm{H},{ }^{3} \mathrm{~J}=8.7 \mathrm{~Hz}\right), 9.38(\mathrm{~s}, 1 \mathrm{H}) ;{ }^{13} \mathrm{C}$ NMR $\left(\mathrm{CDCl}_{3}, 75 \mathrm{MHz}\right): \delta=47.6,111.7,120.5,125.6,125.8,126.8,127.2$, 127.8, 127.9, 128.1, 128.4, 128.5, 128.6, 129.2, 129.3, 129.7, 132.1, 132.3, 135.2, 135.6, 137.6, 149.1, 149.5, 157.4, 178.7; IR (ATR, $\mathrm{cm}^{-1}$ ): $\nu=2964(\mathrm{w}), 1616(\mathrm{~s}), 1552(\mathrm{~m}), 1499(\mathrm{w}), 1410(\mathrm{~m}), 1328(\mathrm{~m}), 1200$ (w), 1147 (m), 1088 (w), 850 (w), 832 (s), 611 (m); GC-MS (EI, $70 \mathrm{eV}): m / z(\%)=397(43), 396\left(\mathrm{M}^{+}, 51\right), 395$ (100), 361 (19), 283 (15), 125 (18); HRMS (ESI-TOF): calcd for $\mathrm{C}_{25} \mathrm{H}_{17} \mathrm{ClN}_{2} \mathrm{O}[\mathrm{M}+\mathrm{H}]^{+}$ 397.11022, found 397.11104. Anal. Calcd for $\mathrm{C}_{25} \mathrm{H}_{16} \mathrm{ClN}_{2} \mathrm{O}$ (396.868): C, 75.66; H, 4.32; N, 7.06. Found: C, 75.98, H, 4.46; N, 7.06.

\subsection{1-(3-(1H-Imidazol-1-yl)propyl)-2-phenylbenzo[b][1,8] naphthyridin-4-(1H)-one (31)}

Starting with 1-(2-chloroquinolin-3-yl)-3-phenylprop-2-yn-1one 2 (170 $\mathrm{mg}, 0.58 \mathrm{mmol}$ ), $N$-(3-aminopropyl)imidazole ( $88 \mathrm{mg}$, $0.7 \mathrm{mmol})$, potassium carbonate $(162 \mathrm{mg}, 1.17 \mathrm{mmol}), \mathrm{Pd}\left(\mathrm{PPh}_{3}\right)_{4}$ (5 mol \%), and DMF (10 ml) were refluxed at $170{ }^{\circ} \mathrm{C}$ for $3 \mathrm{~h}, 31$ was isolated after column chromatography (silica gel, $n$-heptane/ EtOAc $=2: 1)$ as a yellow solid $(135 \mathrm{mg}, 70 \%), \mathrm{mp} 229{ }^{\circ} \mathrm{C} .{ }^{1} \mathrm{H}$ NMR $\left(\mathrm{CDCl}_{3}, 250 \mathrm{MHz}\right): \delta=2.19(\mathrm{~m}, 2 \mathrm{H}), 3.90\left(\mathrm{dd}, 2 \mathrm{H},{ }^{3} \mathrm{~J}=6.3 \mathrm{~Hz}\right.$, $\left.{ }^{4} J=2.4 \mathrm{~Hz}\right), 4.41\left(\mathrm{dd}, 2 \mathrm{H},{ }^{3} \mathrm{~J}=7.5 \mathrm{~Hz},{ }^{4} \mathrm{~J}=3.1 \mathrm{~Hz}\right), 6.18(\mathrm{~s}, 1 \mathrm{H}), 6.76(\mathrm{~s}$, 1H), $7.02(\mathrm{~s}, 1 \mathrm{H}), 7.41(\mathrm{~m}, 3 \mathrm{H}), 7.54(\mathrm{~m}, 4 \mathrm{H}), 7.84(\mathrm{~m}, 1 \mathrm{H}), 8.01$ (dd, $\left.2 \mathrm{H},{ }^{3} \mathrm{~J}=7.3 \mathrm{~Hz},{ }^{4} \mathrm{~J}=3.2 \mathrm{~Hz}\right), 9.28(\mathrm{~s}, 1 \mathrm{H}) ;{ }^{13} \mathrm{C} \mathrm{NMR}\left(\mathrm{CDCl}_{3}, 75 \mathrm{MHz}\right)$ : $\delta=30.2,43.8,44.5,111.4,120.6,125.4,125.8,127.9,127.9,128.0$, 129.0, 129.1, 129.3, 129.9, 132.5, 135.4, 137.7, 137.8, 137.9, 149.0, 149.1, 156.5, 156.5, 178.5; IR $\left(\mathrm{KBr}, \mathrm{cm}^{-1}\right): \nu=2921(\mathrm{w}), 1660(\mathrm{~m}), 1631(\mathrm{~m})$, $1582(\mathrm{~s}), 1486(\mathrm{w}), 1349(\mathrm{~m}), 1274(\mathrm{~m}), 1158(\mathrm{~m}), 1092(\mathrm{~m}), 976(\mathrm{~m})$, 847 (m), 698 (s); GC-MS (EI, $70 \mathrm{eV}): m / z(\%)=380\left(\mathrm{M}^{+}, 28\right), 299$ (13), 285 (100), 273 (18), 272 (17), 207 (25); HRMS (ESI-TOF): calcd for $\mathrm{C}_{24} \mathrm{H}_{21} \mathrm{~N}_{4} \mathrm{O}[\mathrm{M}+\mathrm{H}]^{+} 381.17099$, found 381.17188. Anal. Calcd for $\mathrm{C}_{24} \mathrm{H}_{20} \mathrm{~N}_{4} \mathrm{O}$ (380.442): C, 75.77; H, 5.30; N, 14.73. Found: C, 75.98; H, $5.46 ; \mathrm{N}, 14.66$.

\subsection{1-(4-Methoxyphenyl)-2-phenylbenzo[b][1,8]naphthyr- idin-4(1H)-one (3m)}

Starting with 1-(2-chloroquinolin-3-yl)-3-phenylprop-2-yn-1one 2 (170 mg, $0.58 \mathrm{mmol}), \quad p$-methoxyaniline $(76 \mathrm{mg}$, $0.62 \mathrm{mmol}$ ), $\mathrm{Cs}_{2} \mathrm{CO}_{3}$ (235 mg, $0.72 \mathrm{mmol}$ ), $\mathrm{Pd}\left(\mathrm{PPh}_{3}\right)_{4}(5 \mathrm{~mol} \%$ ), and toluene $\left(10 \mathrm{ml}\right.$ ) were refluxed at $90{ }^{\circ} \mathrm{C}$ for $6 \mathrm{~h}, 3 \mathrm{~m}$ was isolated after column chromatography (silica gel, $n$-heptane/EtOAc $=1: 1$ ) as a yellow solid (142 mg, 73\%), $\mathrm{mp} 290{ }^{\circ} \mathrm{C} .{ }^{1} \mathrm{H} \mathrm{NMR}\left(\mathrm{CDCl}_{3}, 300 \mathrm{MHz}\right)$ : $\delta=3.81(\mathrm{~s}, 3 \mathrm{H}), 6.43(\mathrm{~s}, 1 \mathrm{H}), 6.84\left(\mathrm{~d}, 2 \mathrm{H},{ }^{3} \mathrm{~J}=9.0 \mathrm{~Hz}\right), 7.12(\mathrm{~d}, 2 \mathrm{H}$, $\left.{ }^{3} \mathrm{~J}=9.0 \mathrm{~Hz}\right), 7.26(\mathrm{~m}, 5 \mathrm{H}), 7.51\left(\mathrm{t}, 1 \mathrm{H},{ }^{3} \mathrm{~J}=8.9 \mathrm{~Hz}\right), 7.72(\mathrm{t}, 1 \mathrm{H}$, $\left.{ }^{3} \mathrm{~J}=8.9 \mathrm{~Hz}\right), 7.82\left(\mathrm{~d}, 1 \mathrm{H},{ }^{3} \mathrm{~J}=6.2 \mathrm{~Hz}\right), 8.04\left(\mathrm{~d}, 1 \mathrm{H},{ }^{3} \mathrm{~J}=9.0 \mathrm{~Hz}\right), 9.38(\mathrm{~s}$, $1 \mathrm{H}) ;{ }^{13} \mathrm{C}$ NMR $\left(\mathrm{CDCl}_{3}, 75 \mathrm{MHz}\right): \delta=55.4,111.3,113.6,113.7,120.2$, 125.6, 125.7, 128.0, 128.6, 128.7, 129.0, 129.0, 129.1, 131.3, 131.3, 131.9, 131.9, 136.1, 137.3, 149.1, 150.9, 157.1, 158.8, 179.2; IR (ATR, $\mathrm{cm}^{-1}$ ): $\nu=2921(\mathrm{w}), 1660(\mathrm{~m}), 1631(\mathrm{~m}), 1582(\mathrm{~s}), 1486(\mathrm{w}), 1349(\mathrm{~m}), 1274$ (m), $1158(\mathrm{~m}), 1092(\mathrm{~m}), 976(\mathrm{~m}), 847$ (m), 698 (s); GC-MS (EI, $70 \mathrm{eV}): m / z(\%)=379(14), 378\left(\mathrm{M}^{+}, 65\right), 377$ (100); HRMS (ESI-TOF): calcd for $\mathrm{C}_{25} \mathrm{H}_{19} \mathrm{~N}_{2} \mathrm{O}_{2}[\mathrm{M}+\mathrm{H}]^{+}$379.14410, found 379.14384. Anal. Calcd for $\mathrm{C}_{25} \mathrm{H}_{18} \mathrm{~N}_{2} \mathrm{O}_{2}$ (378.423): C, 79.35; $\mathrm{H}, 4.79$; N, 7.40. Found: $\mathrm{C}$, 78.98; H, 4.69; N, 7.40.

\subsection{1-(3,4-Dimethoxyphenyl)-2-phenylbenzo[b][1,8]naph- thyridin-4(1H)-one (3n)}

Starting with 1-(2-chloroquinolin-3-yl)-3-phenylprop-2-yn-1one 2 (170 $\mathrm{mg}, 0.58 \mathrm{mmol}$ ), 3,4-dimethoxyaniline (95 $\mathrm{mg}$, $0.62 \mathrm{mmol}$ ), $\mathrm{Cs}_{2} \mathrm{CO}_{3}$ (235 mg, $\left.0.72 \mathrm{mmol}\right), \mathrm{Pd}\left(\mathrm{PPh}_{3}\right)_{4}(5 \mathrm{~mol} \%$ ), and toluene $(10 \mathrm{ml})$ were refluxed at $90{ }^{\circ} \mathrm{C}$ for $6 \mathrm{~h}, 3 \mathrm{n}$ was isolated after column chromatography (silica gel, $n$-heptane/EtOAc $=1: 1$ ) as a yellow solid (150 mg, 76\%), mp $235^{\circ} \mathrm{C} .{ }^{1} \mathrm{H} \mathrm{NMR}\left(\mathrm{CDCl}_{3}, 300 \mathrm{MHz}\right)$ : $\delta=3.66(\mathrm{~s}, 3 \mathrm{H}), 3.81(\mathrm{~s}, 3 \mathrm{H}), 6.35(\mathrm{~s}, 1 \mathrm{H}), 6.66(\mathrm{~s}, 1 \mathrm{H}), 6.71(\mathrm{~s}, 2 \mathrm{H}), 7.19$ $(\mathrm{m}, 5 \mathrm{H}), 7.43\left(\mathrm{t}, 1 \mathrm{H},{ }^{3} \mathrm{~J}=7.5 \mathrm{~Hz}\right), 7.65\left(\mathrm{t}, 1 \mathrm{H},{ }^{3} \mathrm{~J}=8.3 \mathrm{~Hz}\right), 7.75(\mathrm{~d}, 1 \mathrm{H}$, $\left.{ }^{3} \mathrm{~J}=9.0 \mathrm{~Hz}\right), 7.95\left(\mathrm{~d}, 1 \mathrm{H},{ }^{3} \mathrm{~J}=8.8 \mathrm{~Hz}\right), 9.25(\mathrm{~s}, 1 \mathrm{H}) ;{ }^{13} \mathrm{C} \mathrm{NMR}\left(\mathrm{CDCl}_{3}\right.$, $75 \mathrm{MHz}): \delta=55.9,56.1,110.3,111.4,114.0,120.2,122.9,125.6,125.7$, 128.0, 128.2, 128.2, 128.6, 128.7, 128.8, 129.0, 129.1, 131.9, 136.2, 137.3, 148.4, 148.4, 148.7, 149.1, 150.8, 157.1, 179.1; IR (ATR, $\mathrm{cm}^{-1}$ ): $\nu=2833(\mathrm{w}), 1633(\mathrm{~m}), 1509(\mathrm{~m}), 1471(\mathrm{~m}), 1398(\mathrm{~s}), 1255$ (s), 1208 (m), $1132(\mathrm{~m}), 1026(\mathrm{~s}), 841(\mathrm{~m}), 759(\mathrm{~s}), 705(\mathrm{~s})$; GC-MS (EI, $70 \mathrm{eV})$ : $m / z(\%)=409$ (19), $408\left(\mathrm{M}^{+}, 78\right), 407$ (100), 391 (12), 321 (14); HRMS (ESI-TOF): calcd for $\mathrm{C}_{26} \mathrm{H}_{21} \mathrm{~N}_{2} \mathrm{O}_{3} \quad[\mathrm{M}+\mathrm{H}]^{+}$409.15467, found 409.15504. Anal. Calcd for $\mathrm{C}_{26} \mathrm{H}_{20} \mathrm{~N}_{2} \mathrm{O}_{3}$ (408.449): C, 76.45; $\mathrm{H}, 4.94$; N, 6.86. Found: C, 75.97; H, 4.91; N, 6.84.

\subsection{1-(3,5-Dimethoxyphenyl)-2-phenylbenzo[b][1,8]naph- thyridin-4(1H)-one (3o)}

Starting with 1-(2-chloroquinolin-3-yl)-3-phenylprop-2-yn-1one 2 (170 mg, $0.58 \mathrm{mmol}$ ), 3,5-dimethoxyaniline (95 mg, $0.62 \mathrm{mmol}), \mathrm{Cs}_{2} \mathrm{CO}_{3}(235 \mathrm{mg}, 0.72 \mathrm{mmol}), \mathrm{Pd}\left(\mathrm{PPh}_{3}\right)_{4}(5 \mathrm{~mol} \%)$, and toluene $(10 \mathrm{ml})$ were refluxed at $90^{\circ} \mathrm{C}$ for $6 \mathrm{~h}, 3 \mathrm{o}$ was isolated after column chromatography (silica gel, $n$-heptane/EtOAc $=1: 1$ ) as a yellow solid (135 mg, 64\%), $\mathrm{mp} 239^{\circ} \mathrm{C} .{ }^{1} \mathrm{H} \mathrm{NMR}\left(\mathrm{CDCl}_{3}, 300 \mathrm{MHz}\right)$ : $\delta=3.58(\mathrm{~s}, 6 \mathrm{H}), 6.30(\mathrm{~m}, 4 \mathrm{H}), 7.19(\mathrm{~m}, 5 \mathrm{H}), 7.41\left(\mathrm{t}, 1 \mathrm{H},{ }^{3} \mathrm{~J}=7.6 \mathrm{~Hz}\right), 7.63$ $\left(\mathrm{t}, 1 \mathrm{H},{ }^{3} \mathrm{~J}=7.8 \mathrm{~Hz}\right), 7.76\left(\mathrm{~d}, 1 \mathrm{H},{ }^{3} \mathrm{~J}=8.7 \mathrm{~Hz}\right), 7.93\left(\mathrm{~d}, 1 \mathrm{H},{ }^{3} \mathrm{~J}=7.3 \mathrm{~Hz}\right), 9.22$ (s, $1 \mathrm{H}) ;{ }^{13} \mathrm{C} \mathrm{NMR}\left(\mathrm{CDCl}_{3}, 75 \mathrm{MHz}\right): \delta=55.5,55.6,100.4,109.3,109.4$, $111.4,120.0,120.2,125.6,125.7,127.9,128.0,128.7,128.9,128.9$, 129.1, 131.9, 136.1, 137.3, 140.5, 149.0, 149.1, 150.4, 156.6, 160.4, 179.1; IR (ATR, $\left.\mathrm{cm}^{-1}\right): \nu=3053(\mathrm{w}), 1637(\mathrm{~m}), 1582(\mathrm{~s}), 1403(\mathrm{~m}), 1316(\mathrm{~m})$, 1150 (s), 1060 (m), 950 (w), $826(\mathrm{~m}), 773(\mathrm{~m}), 705(\mathrm{~m}), 613(\mathrm{w})$; GC-MS (EI, $70 \mathrm{eV}): m / z(\%)=409$ (15), $408\left(\mathrm{M}^{+}, 66\right), 407$ (100), 153 (10); HRMS (ESI-TOF): calcd for $\mathrm{C}_{26} \mathrm{H}_{21} \mathrm{~N}_{2} \mathrm{O}_{3}[\mathrm{M}+\mathrm{H}]^{+}$409.15467, found 409.15580. Anal. Calcd for $\mathrm{C}_{26} \mathrm{H}_{20} \mathrm{~N}_{2} \mathrm{O}_{3}$ (408.449): C, 76.45; $\mathrm{H}$, 4.94; N, 6.86. Found: C, 76.37; H, 4.89; N, 6.85.

\subsection{2-Phenyl-1-(3,4,5-trimethoxyphenyl)benzo[b][1,8]naph- thyridin-4(1H)-one (3p)}

Starting with 1-(2-chloroquinolin-3-yl)-3-phenylprop-2-yn-1one 2 (170 mg, $0.58 \mathrm{mmol}$ ), 3,4,5-trimethoxyaniline (113 mg, $0.62 \mathrm{mmol}$ ), $\mathrm{Cs}_{2} \mathrm{CO}_{3}$ (235 mg, $0.72 \mathrm{mmol}$ ), $\mathrm{Pd}\left(\mathrm{PPh}_{3}\right)_{4}(5 \mathrm{~mol} \%$ ), and toluene $(10 \mathrm{ml})$ were refluxed at $90{ }^{\circ} \mathrm{C}$ for $6 \mathrm{~h}, 3 \mathrm{p}$ was isolated after column chromatography (silica gel, $n$-heptane/EtOAc $=1: 1$ ) as a yellow solid (173 mg, 77\%), mp $253{ }^{\circ} \mathrm{C} .{ }^{1} \mathrm{H} \mathrm{NMR}\left(\mathrm{CDCl}_{3}, 300 \mathrm{MHz}\right)$ : $\delta=3.62(\mathrm{~s}, 6 \mathrm{H}), 3.78(\mathrm{~s}, 3 \mathrm{H}), 6.35(\mathrm{~s}, 1 \mathrm{H}), 6.39(\mathrm{~s}, 2 \mathrm{H}), 7.22(\mathrm{~m}, 5 \mathrm{H})$, $7.44\left(\mathrm{t}, 1 \mathrm{H},{ }^{3} \mathrm{~J}=7.6 \mathrm{~Hz}\right), 7.67\left(\mathrm{t}, 1 \mathrm{H},{ }^{3} \mathrm{~J}=7.6 \mathrm{~Hz}\right), 7.80\left(\mathrm{~d}, 1 \mathrm{H},{ }^{3} \mathrm{~J}=9.1 \mathrm{~Hz}\right)$, $7.96\left(\mathrm{~d}, 1 \mathrm{H},{ }^{3} \mathrm{~J}=9.1 \mathrm{~Hz}\right), 9.26(\mathrm{~s}, 1 \mathrm{H}) ;{ }^{13} \mathrm{C} \mathrm{NMR}\left(\mathrm{CDCl}_{3}, 75 \mathrm{MHz}\right)$ : $\delta=56.3,61.1,108.6,111.4,120.1,125.6,125.7,125.8,128.3,128.5$, 128.6, 128.9, 129.0, 129.2, 129.4, 132.1, 134.4, 136.2, 137.4, 137.7, 149.1, 150.5, 152.9, 156.8, 179.1; IR (ATR, $\mathrm{cm}^{-1}$ ): $\nu=2964(\mathrm{w}), 1637(\mathrm{~m})$, $1556(\mathrm{w}), 1495$ (m), $1404(\mathrm{~m}), 1227(\mathrm{~s}), 949(\mathrm{w}), 854(\mathrm{w}), 825(\mathrm{~m})$, $712(\mathrm{~m}), 643(\mathrm{~m}) ; \mathrm{GC}-\mathrm{MS}(\mathrm{EI}, 70 \mathrm{eV}): \mathrm{m} / z(\%)=439(28), 438\left(\mathrm{M}^{+}\right.$, 100), 437 (63), 423 (21), 392 (13), 391 (20); HRMS (ESI-TOF): calcd for $\mathrm{C}_{27} \mathrm{H}_{23} \mathrm{~N}_{2} \mathrm{O}_{4}[\mathrm{M}+\mathrm{H}]^{+}$439.16523, found 439.16492. Anal. Calcd for $\mathrm{C}_{27} \mathrm{H}_{22} \mathrm{~N}_{2} \mathrm{O}_{4}$ (438.475): C, 73.96; $\mathrm{H}, 5.06 ; \mathrm{N}, 6.39$. Found: C, 73.37; H, 4.99; N, 6.35. 
5.19. 2-Phenyl-1-(3,5-dimethylphenyl)benzo[b] $[1,8]$ naphthyridin-4(1H)-one (3q)

Starting with 1-(2-chloroquinolin-3-yl)-3-phenylprop-2-yn-1one 2 (170 $\mathrm{mg}, 0.58 \mathrm{mmol}), 3,5$-dimethylaniline (75 $\mathrm{mg}$, $0.62 \mathrm{mmol}), \mathrm{Cs}_{2} \mathrm{CO}_{3}(235 \mathrm{mg}, 0.72 \mathrm{mmol}), \mathrm{Pd}\left(\mathrm{PPh}_{3}\right)_{4}(5 \mathrm{~mol} \%)$, and toluene $(10 \mathrm{ml})$ were refluxed at $90{ }^{\circ} \mathrm{C}$ for $6 \mathrm{~h}, \mathbf{3 q}$ was isolated after column chromatography (silica gel, $n$-heptane/EtOAc $=2: 1$ ) as a yellow solid (128 mg, 58\%), mp $255{ }^{\circ} \mathrm{C} .{ }^{1} \mathrm{H} \mathrm{NMR}\left(\mathrm{CDCl}_{3}\right.$, $300 \mathrm{MHz}): \delta=2.27(\mathrm{~s}, 6 \mathrm{H}), 6.45(\mathrm{~s}, 1 \mathrm{H}), 6.85(\mathrm{~s}, 2 \mathrm{H}), 6.91(\mathrm{~s}, 1 \mathrm{H}), 7.27$ (s, $5 \mathrm{H}), 7.53\left(\mathrm{t}, 1 \mathrm{H},{ }^{3} \mathrm{~J}=6.7 \mathrm{~Hz}\right), 7.74\left(\mathrm{t}, 1 \mathrm{H},{ }^{3} \mathrm{~J}=6.8 \mathrm{~Hz}\right), 7.85(\mathrm{~d}, 1 \mathrm{H}$, $\left.{ }^{3} \mathrm{~J}=9.3 \mathrm{~Hz}\right), 8.10\left(\mathrm{~d}, 1 \mathrm{H},{ }^{3} \mathrm{~J}=9.3 \mathrm{~Hz}\right), 9.37(\mathrm{~s}, 1 \mathrm{H}) ;{ }^{13} \mathrm{C}$ NMR $\left(\mathrm{CDCl}_{3}\right.$, $75 \mathrm{MHz}$ ): $\delta=21.1,111.2,120.1,125.5,125.6,127.8,128.0,128.1,128.6$, 128.7, 128.7, 128.8, 128.9, 129.1, 129.5, 131.9, 136.1, 137.3, 138.0, 138.1, 138.8, 149.1, 150.7, 157.0, 179.9; IR (ATR, $\left.\mathrm{cm}^{-1}\right): \nu=3057(\mathrm{w})$, $1628(\mathrm{~m}), 1550(\mathrm{~m}), 1403(\mathrm{~s}), 1335(\mathrm{~m}), 138(\mathrm{~s}), 1438(\mathrm{~m}), 1148(\mathrm{~m})$, $971(\mathrm{~m}), 840(\mathrm{~m}), 756(\mathrm{~s}), 588(\mathrm{~m})$; GC-MS (EI, $70 \mathrm{eV}): \mathrm{m} / \mathrm{z}(\%)=$ 377 (17), $376\left(\mathrm{M}^{+}, 73\right), 375$ (100), 347 (10); HRMS (ESI-TOF): calcd for $\mathrm{C}_{26} \mathrm{H}_{21} \mathrm{~N}_{2} \mathrm{O}[\mathrm{M}+\mathrm{H}]^{+}$377.16484, found 377.16412. Anal. Calcd for $\mathrm{C}_{26} \mathrm{H}_{20} \mathrm{~N}_{2} \mathrm{O}$ (376.450): C, 82.95; $\mathrm{H}, 5.35 ; \mathrm{N}, 7.44$. Found: $\mathrm{C}, 82.87 ; \mathrm{H}$, 5.29; N, 7.44.

\subsection{2-Phenyl-1-(2,4-dimethoxyphenyl)benzo $[b][1,8]$ naph- thyridin-4(1H)-one (3r)}

Starting with 1-(2-chloroquinolin-3-yl)-3-phenylprop-2-yn-1one 2 (170 mg, $0.58 \mathrm{mmol}$ ), 3,5-dimethoxyaniline (126 mg, $0.62 \mathrm{mmol}$ ), $\mathrm{Cs}_{2} \mathrm{CO}_{3}$ (235 mg, $0.72 \mathrm{mmol}$ ), $\mathrm{Pd}\left(\mathrm{PPh}_{3}\right)_{4}(5 \mathrm{~mol} \%)$, and toluene $(10 \mathrm{ml})$ were refluxed at $90{ }^{\circ} \mathrm{C}$ for $6 \mathrm{~h}, 3 \mathrm{r}$ was isolated after column chromatography (silica gel, $n$-heptane/EtOAc $=2: 1$ ) as a yellow solid (126 mg, 53\%), $\mathrm{mp} 275^{\circ} \mathrm{C} .{ }^{1} \mathrm{H}$ NMR $\left(\mathrm{CDCl}_{3}, 300 \mathrm{MHz}\right)$ : $\delta=3.52(\mathrm{~s}, 3 \mathrm{H}), 3.75(\mathrm{~s}, 3 \mathrm{H}), 6.35(\mathrm{~m}, 3 \mathrm{H}), 7.03\left(\mathrm{~d}, 1 \mathrm{H},{ }^{3} \mathrm{~J}=7.3 \mathrm{~Hz}\right), 7.20$ $(\mathrm{m}, 5 \mathrm{H}), 7.64\left(\mathrm{t}, 1 \mathrm{H},{ }^{3} \mathrm{~J}=7.3 \mathrm{~Hz}\right), 7.43\left(\mathrm{t}, 1 \mathrm{H},{ }^{3} \mathrm{~J}=6.4 \mathrm{~Hz}\right), 7.74(\mathrm{~d}, 1 \mathrm{H}$, $\left.{ }^{3} \mathrm{~J}=8.3 \mathrm{~Hz}\right), 7.97\left(\mathrm{~d}, 1 \mathrm{H},{ }^{3} \mathrm{~J}=8.3 \mathrm{~Hz}\right), 9.27(\mathrm{~s}, 1 \mathrm{H}) ;{ }^{13} \mathrm{C} \mathrm{NMR}\left(\mathrm{CDCl}_{3}\right.$, $75 \mathrm{MHz}): \delta=55.4,55.5,99.0,104.1,110.8,120.3,121.6,125.4,125.6$, 127.6, 127.7, 128.4, 128.5, 128.6, 128.8, 129.1, 131.7, 131.8, 136.1, 137.1, 149.3, 150.6, 156.2, 157.8, 160.7, 179.5; IR (ATR, $\left.\mathrm{cm}^{-1}\right): \nu=2921(\mathrm{w})$, $1660(\mathrm{~m}), 1631(\mathrm{~m}), 1582(\mathrm{~s}), 1486(\mathrm{w}), 1349(\mathrm{~m}), 1274(\mathrm{~m}), 1158(\mathrm{~m})$, $1092(\mathrm{~m}), 976(\mathrm{~m}), 847$ (m), 698 (s); GC-MS (EI, $70 \mathrm{eV}): \mathrm{m} / \mathrm{z}(\%)=$ $408\left(\mathrm{M}^{+}, 3\right), 378$ (28), 377 (100); HRMS (ESI-TOF): calcd for $\mathrm{C}_{26} \mathrm{H}_{21} \mathrm{~N}_{2} \mathrm{O}_{3}[\mathrm{M}+\mathrm{H}]^{+}$409.15467, found 409.15496. Anal. Calcd for $\mathrm{C}_{26} \mathrm{H}_{20} \mathrm{~N}_{2} \mathrm{O}_{3}$ (408.449): C, 76.45; H, 4.94; N, 6.86. Found: C, 76.63; H, $5.05 ; \mathrm{N}, 6.98$.

\subsection{1-(4-(Diethylamino)phenyl)-2-phenylbenzo $[b][1,8]$ naphthyridin-4(1H)-one (3s)}

Starting with 1-(2-chloroquinolin-3-yl)-3-phenylprop-2-yn-1one 2 (170 mg, $0.58 \mathrm{mmol}), N, N$-dimethylbenzene-1,4-diamine (112 mg, $0.62 \mathrm{mmol}), \mathrm{Cs}_{2} \mathrm{CO}_{3}(235 \mathrm{mg}, 0.72 \mathrm{mmol}), \mathrm{Pd}\left(\mathrm{PPh}_{3}\right)_{4}$ ( $5 \mathrm{~mol} \%)$, and toluene $\left(10 \mathrm{ml}\right.$ ) were refluxed at $90^{\circ} \mathrm{C}$ for $6 \mathrm{~h}, 3 \mathrm{~s}$ was isolated after column chromatography (silica gel, $n$-heptane/ EtOAc $=3: 1$ ) as a yellow solid (167 mg, 77\%), mp $320{ }^{\circ} \mathrm{C} .{ }^{1} \mathrm{H}$ NMR $\left(\mathrm{CDCl}_{3}, 300 \mathrm{MHz}\right): \delta=1.31\left(\mathrm{t}, 6 \mathrm{H},{ }^{3} \mathrm{~J}=7.6 \mathrm{~Hz}\right), 2.49\left(\mathrm{q}, 4 \mathrm{H},{ }^{3} \mathrm{~J}=7.6 \mathrm{~Hz}\right)$, $5.59(\mathrm{~s}, 1 \mathrm{H}), 5.71\left(\mathrm{~d}, 2 \mathrm{H},{ }^{3} \mathrm{~J}=8.0 \mathrm{~Hz}\right), 6.14\left(\mathrm{~d}, 2 \mathrm{H},{ }^{3} \mathrm{~J}=8.7 \mathrm{~Hz}\right), 6.42(\mathrm{~s}$, $5 \mathrm{H}), 6.66\left(\mathrm{t}, 1 \mathrm{H},{ }^{3} \mathrm{~J}=7.7 \mathrm{~Hz}\right), 6.87\left(\mathrm{t}, 1 \mathrm{H},{ }^{3} \mathrm{~J}=7.7 \mathrm{~Hz}\right), 7.04(\mathrm{~d}, 1 \mathrm{H}$, $\left.{ }^{3} \mathrm{~J}=8.0 \mathrm{~Hz}\right), 7.19\left(\mathrm{~d}, 1 \mathrm{H},{ }^{3} \mathrm{~J}=7.2 \mathrm{~Hz}\right), 8.5(\mathrm{~s}, 1 \mathrm{H}) ;{ }^{13} \mathrm{C} \mathrm{NMR}\left(\mathrm{CDCl}_{3}\right.$, $75 \mathrm{MHz}): \delta=12.4,44.4,111.1,111.2,120.3,125.5,127.1,127.8,127.9$, $128.5,128.7,129.0,129.1,129.2,130.7,130.8,131.7,136.5,136.6,137.2$, 147.1, 149.2, 151.2, 157.7, 179.2; IR (ATR, $\left.\mathrm{cm}^{-1}\right): \nu=2968$ (w), 1634 (m), $1515(\mathrm{~s}), 1473(\mathrm{~m}), 1262(\mathrm{~m}), 1195(\mathrm{~m}), 1009(\mathrm{w}), 827(\mathrm{~m}), 741$ (s), $701(\mathrm{~m}), 605(\mathrm{~m})$; GC-MS (EI, $70 \mathrm{eV}): \mathrm{m} / \mathrm{z}(\%)=420$ (22), 419 $\left(\mathrm{M}^{+}, 72\right), 418$ (18), 405 (30), 404 (100), 374 (24), 159 (10); HRMS (ESI-TOF): calcd for $\mathrm{C}_{28} \mathrm{H}_{26} \mathrm{~N}_{3} \mathrm{O} \quad[\mathrm{M}+\mathrm{H}]^{+}$420.20704, found 420.20700. Anal. Calcd for $\mathrm{C}_{28} \mathrm{H}_{25} \mathrm{~N}_{3} \mathrm{O}$ (419.518): C, 80.16; $\mathrm{H}, 6.01 ; \mathrm{N}$, 10.02. Found: C, $80.21 ; \mathrm{H}, 6.06 ; \mathrm{N}, 10.02$.

\subsection{1-(4-Ethylphenyl)-2-phenylbenzo[b][1,8]naphthyridin- $4(1 H)$-one (3t)}

Starting with 1-(2-chloroquinolin-3-yl)-3-phenylprop-2-yn-1one 2 (170 $\mathrm{mg}, 0.58 \mathrm{mmol}$ ), p-ethylaniline ( $75 \mathrm{mg}, 0.62 \mathrm{mmol}$ ), $\mathrm{Cs}_{2} \mathrm{CO}_{3}$ (235 mg, $\left.0.72 \mathrm{mmol}\right), \mathrm{Pd}\left(\mathrm{PPh}_{3}\right)_{4}(5 \mathrm{~mol} \%)$, and toluene $\left(10 \mathrm{ml}\right.$ ) were refluxed at $90{ }^{\circ} \mathrm{C}$ for $6 \mathrm{~h}, 3 \mathrm{t}$ was isolated after column chromatography (silica gel, $n$-heptane/EtOAc $=3: 1$ ) as a yellow solid (96 mg, 50\%), mp $229^{\circ} \mathrm{C} .{ }^{1} \mathrm{H} \mathrm{NMR}\left(\mathrm{CDCl}_{3}, 300 \mathrm{MHz}\right): \delta=1.31$ (t, $3 \mathrm{H}$, ${ }^{3} \mathrm{~J}=7.8 \mathrm{~Hz}$ ), $2.55(\mathrm{~m}, 2 \mathrm{H}), 6.34(\mathrm{~s}, 1 \mathrm{H}), 7.02\left(\mathrm{q}, 4 \mathrm{H},{ }^{3} \mathrm{~J}=9.5 \mathrm{~Hz}\right), 7.14(\mathrm{~s}$, $5 \mathrm{H}), 7.4\left(\mathrm{t}, 1 \mathrm{H},{ }^{3} \mathrm{~J}=7.5 \mathrm{~Hz}\right), 7.61\left(\mathrm{t}, 1 \mathrm{H},{ }^{3} \mathrm{~J}=6.8 \mathrm{~Hz}\right), 7.72(\mathrm{~d}, 1 \mathrm{H}$, $\left.{ }^{3} \mathrm{~J}=8.5 \mathrm{~Hz}\right), 7.94\left(\mathrm{~d}, 1 \mathrm{H},{ }^{3} \mathrm{~J}=8.3 \mathrm{~Hz}\right), 9.24(\mathrm{~s}, 1 \mathrm{H}) ;{ }^{3} \mathrm{C} \mathrm{NMR}\left(\mathrm{CDCl}_{3}\right.$, $75 \mathrm{MHz}$ ): $\delta=15.2,28.4,111.3,120.1,125.6,125.7,127.7,127.8,128.6$, 128.7, 128.8, 129.0, 129.1, 129.2, 130.2, 130.3, 131.9, 131.9, 136.1, 136.7, 137.3, 143.9, 149.1, 150.8, 157.0, 179.3; IR (ATR, $\left.\mathrm{cm}^{-1}\right): \nu=3029(\mathrm{w})$, $3004(\mathrm{w}), 1637(\mathrm{~m}), 1473(\mathrm{~m}), 1184(\mathrm{w}), 1062(\mathrm{w}), 828(\mathrm{~s}), 778(\mathrm{~m})$, 739 (m), 560 (m), 545 (w); GC-MS (EI, $70 \mathrm{eV}): \mathrm{m} / z(\%)=377$ (12), $376\left(\mathrm{M}^{+}, 54\right), 375$ (100); HRMS (ESI-TOF): calcd for $\mathrm{C}_{26} \mathrm{H}_{21} \mathrm{~N}_{2} \mathrm{O}$ $[\mathrm{M}+\mathrm{H}]^{+}$377.16484, found 377.16561. Anal. Calcd for $\mathrm{C}_{26} \mathrm{H}_{20} \mathrm{~N}_{2} \mathrm{O}$ (376.450): C, 82.95; H, 5.35; N, 7.44. Found: C, 82.92; H, 5.26; N, 7.43 .

\subsection{1-(2-Fluorophenyl)-2-phenylbenzo $[b][1,8]$ naphthyridin- 4(1H)-one (3u)}

Starting with 1-(2-chloroquinolin-3-yl)-3-phenylprop-2-yn-1one 2 (170 mg, $0.58 \mathrm{mmol}$ ), p-methoxyaniline (76 mg, $0.62 \mathrm{mmol}$ ), $\mathrm{Cs}_{2} \mathrm{CO}_{3}$ (235 mg, $\left.0.72 \mathrm{mmol}\right), \mathrm{Pd}\left(\mathrm{PPh}_{3}\right)_{4}(5 \mathrm{~mol} \%)$, and toluene $(10 \mathrm{ml})$ were refluxed at $90{ }^{\circ} \mathrm{C}$ for $6 \mathrm{~h}, 3 \mathbf{u}$ was isolated after column chromatography (silica gel, $n$-heptane/EtOAc $=1: 1$ ) as a yellow solid (62 mg, 29\%), $\mathrm{mp} 243{ }^{\circ} \mathrm{C} .{ }^{1} \mathrm{H} \mathrm{NMR}\left(\mathrm{CDCl}_{3}, 300 \mathrm{MHz}\right)$ : $\delta=6.36(\mathrm{~s}, 1 \mathrm{H}), 7.04(\mathrm{~m}, 3 \mathrm{H}), 7.18(\mathrm{~m}, 7 \mathrm{H}), 7.45(\mathrm{~m}, 1 \mathrm{H}), 7.68(\mathrm{~m}, 2 \mathrm{H})$, $7.97\left(\mathrm{~d}, 1 \mathrm{H},{ }^{3} \mathrm{~J}=8.4 \mathrm{~Hz}\right), 9.26(\mathrm{~s}, 1 \mathrm{H}) ;{ }^{19} \mathrm{~F} \mathrm{NMR}\left(\mathrm{CDCl}_{3}, 282 \mathrm{MHz}\right)$ : $\delta=-118.4 ;{ }^{13} \mathrm{C}$ NMR (75 MHz, $\mathrm{CDCl}_{3}$ ): $\delta=111.3,115.7,116.1,120.0$, 124.1, 124.2, 126.1 (d, $J=16 \mathrm{~Hz}$ ), 126.3 (d, $J=230 \mathrm{~Hz}$ ), 128.1, 128.4, $128.5,129.1,129.2,129.3,129.4,130.2,131.7,131.8,133.5$ (d, $J=10 \mathrm{~Hz}$ ), 135.1 (d, $J=10 \mathrm{~Hz}), 136.3$ (d, $J=40 \mathrm{~Hz}), 137.4,137.5,149.2$, 179.4; IR (ATR, $\mathrm{cm}^{-1}$ ): $\nu=2919(\mathrm{w}), 1581$ (s), $1554(\mathrm{~m}), 1209(\mathrm{~m})$, 1156 (w), 1031 (m), 950 (m), 851 (w), 774 (s), 562 (m); GC-MS (EI, $70 \mathrm{eV}): m / z(\%)=367$ (13), $366\left(\mathrm{M}^{+}, 52\right), 365$ (16), 348 (26), 347 (100), 346 (7); HRMS (ESI-TOF): calcd for $\mathrm{C}_{24} \mathrm{H}_{16} \mathrm{FN}_{2} \mathrm{O}[\mathrm{M}+\mathrm{H}]^{+}$ 367.12412, found 367.12471. Anal. Calcd for $\mathrm{C}_{24} \mathrm{H}_{15} \mathrm{FN}_{2} \mathrm{O}$ (366.387): C, 78.68; H, 4.13; N, 7.65. Found: C, 78.38; H, 4.10; N, 7.62.

\subsection{1-(3-Chloroquinoxalin-2-yl)-3-phenylprop-2-yn-1-one (5a)}

Yield $3.10 \mathrm{~g}$ (53\%), pale green crystals, mp $81-83{ }^{\circ} \mathrm{C} .{ }^{1} \mathrm{H}$ NMR $\left(\mathrm{CDCl}_{3}, 300 \mathrm{MHz}\right): \delta=7.34-7.48(\mathrm{~m}, 3 \mathrm{H}), 7.63-7.66(\mathrm{~m}, 2 \mathrm{H})$, $7.79-7.90(\mathrm{~m}, 2 \mathrm{H}), 8.03-8.06(\mathrm{~m}, 1 \mathrm{H}), 8.19-8.23(\mathrm{~m}, 1 \mathrm{H}) ;{ }^{13} \mathrm{C} \mathrm{NMR}$ $\left(\mathrm{CDCl}_{3}, 63 \mathrm{MHz}\right): \delta=88.3,97.0,119.7,128.3,128.7,130.1,131.1,131.3$, 133.4, 133.5, 139.8, 142.5, 144.0, 146.4, 175.3; IR (ATR, $\left.\mathrm{cm}^{-1}\right)$ : $\nu=3290(\mathrm{w}), 3105$ (w), $3068(\mathrm{w}), 3045$ (w), $2992(\mathrm{w}), 2956(\mathrm{w})$, 2926 (w), 2872 (w), 2855 (w), 2191 (s), 1725 (w), 1652 (s), 1592 (m), $1572(\mathrm{w}), 1557(\mathrm{~m}), 1536(\mathrm{~m}), 1505(\mathrm{w}), 1485(\mathrm{~m}), 1461(\mathrm{~m})$, 1441 (m), 1418 (w), 1382 (w), 1347 (m), 1321 (m), 1305 (m), 1286 (m), $1260(\mathrm{~m}), 1228(\mathrm{w}), 1212(\mathrm{w}), 1178(\mathrm{w}), 1147(\mathrm{~m}), 1132(\mathrm{~m})$, 1080 (s), 1025 (m), $1002(\mathrm{w}) ; \mathrm{UV}\left(\mathrm{CH}_{2} \mathrm{Cl}_{2}, \mathrm{~nm}\right): \lambda_{\max }(\log \varepsilon)=235$ (5.44), 248 (5.40), 309 (5.09); MS (EI, $70 \mathrm{eV}): m / z(\%)=294\left(\mathrm{M}^{+}\right.$, $\left.{ }^{37} \mathrm{Cl}, 10\right), 292\left(\mathrm{M}^{+},{ }^{35} \mathrm{Cl}, 29\right), 264$ (22), 229 (31), 129 (100), 102 (14), 75 (21), 51 (8); HRMS (ESI): calcd for $\mathrm{C}_{17} \mathrm{H}_{10}^{35} \mathrm{ClN}_{2} \mathrm{O}[\mathrm{M}+\mathrm{H}]^{+}$ 293.04762, found 293.04727; calcd for $\mathrm{C}_{17} \mathrm{H}_{10}^{37} \mathrm{ClN}_{2} \mathrm{O}[\mathrm{M}+\mathrm{H}]^{+}$ 295.04519, found 295.04518. Anal. Calcd for $\mathrm{C}_{17} \mathrm{H}_{9} \mathrm{ClN}_{2} \mathrm{O}$ (292.719): C, 69.75; H, 3.10; N, 9.57. Found: C, 69.62; H, 3.09; N, 9.56 . 
5.25. 3-(4-tert-Butylphenyl)-1-(3-chloroquinoxalin-2-yl) prop-2-yn-1-one (5b)

Yield $0.32 \mathrm{~g}$ (58\%), yellow solid, $\mathrm{mp} 110-112{ }^{\circ} \mathrm{C} .{ }^{1} \mathrm{H}$ NMR $\left(\mathrm{CDCl}_{3}\right.$, $300 \mathrm{MHz}): \delta=1.26(\mathrm{~s}, 9 \mathrm{H}), 7.36-7.39(\mathrm{~m}, 2 \mathrm{H}), 7.55-7.58(\mathrm{~m}, 2 \mathrm{H})$, $7.79-7.88(\mathrm{~m}, 2 \mathrm{H}), 7.99-8.03(\mathrm{~m}, 1 \mathrm{H}), 8.16-8.19(\mathrm{~m}, 1 \mathrm{H}) ;{ }^{13} \mathrm{C} N M R$ $\left(\mathrm{CDCl}_{3}, 63 \mathrm{MHz}\right): \delta=31.0,35.2,88.3,97.9,116.6,125.8,128.3,130.1$, 131.0, 133.2, 133.5, 139.8, 142.4, 143.9, 146.7, 155.3, 175.4; IR (ATR, $\left.\mathrm{cm}^{-1}\right): \nu=3282(\mathrm{w}), 3068(\mathrm{w}), 2960(\mathrm{~m}), 2902(\mathrm{w}), 2864(\mathrm{w}), 2181$ (s), $1939(\mathrm{w}), 1646(\mathrm{~s}), 1598(\mathrm{~m}), 1558(\mathrm{~m}), 1539(\mathrm{w}), 1504(\mathrm{~m}), 1481$ (w), $1460(\mathrm{~m}), 1402(\mathrm{w}), 1386(\mathrm{w}), 1361(\mathrm{~m}), 1347(\mathrm{w}), 1328(\mathrm{~m})$, 1299 (w), 1282 (w), 1266 (m), 1210 (w), 1202 (w), 1188 (w), 1144 (m), 1129 (m), 1121 (w), 1108 (w), 1073 (s), 1022 (w), 1013 (m); UV $\left(\mathrm{CH}_{2} \mathrm{Cl}_{2}, \mathrm{~nm}\right): \lambda_{\max }(\log \varepsilon)=228$ (5.78), 254 (5.58), 297 (5.40); MS (EI): $m / z(\%)=350\left(\mathrm{M}^{+},{ }^{37} \mathrm{Cl}, 10\right), 348\left(\mathrm{M}^{+},{ }^{35} \mathrm{Cl}, 30\right), 333$ (19), 305 (21), 277 (6), 185 (100), 170 (22), 155 (27), 115 (8), 102 (19), 51 (3); HRMS (ESI): calcd for $\mathrm{C}_{21} \mathrm{H}_{18} \mathrm{ClN}_{2} \mathrm{O}\left([\mathrm{M}+\mathrm{H}]^{+},{ }^{35} \mathrm{Cl}\right) 349.11022$, found 349.11068; calcd for $\mathrm{C}_{21} \mathrm{H}_{18} \mathrm{ClN}_{2} \mathrm{O}\left([\mathrm{M}+\mathrm{H}]+{ }^{+},{ }^{37} \mathrm{Cl}\right) 351.10803$, found 351.10852 .

\subsection{1-(3-Chloroquinoxalin-2-yl)undec-2-yn-1-one (5c)}

Yield $0.19 \mathrm{~g}$ (35\%), yellow oil. ${ }^{1} \mathrm{H}$ NMR $\left(\mathrm{CDCl}_{3}, 300 \mathrm{MHz}\right)$ : $\delta=0.77-0.82(\mathrm{~m}, 3 \mathrm{H}), 1.20-1.24(\mathrm{~m}, 8 \mathrm{H}), 1.37-1.46(\mathrm{~m}, 2 \mathrm{H})$, $1.56-1.65(\mathrm{~m}, 2 \mathrm{H}), 2.46\left(\mathrm{t}, 2 \mathrm{H},{ }^{3} \mathrm{~J}=7.0 \mathrm{~Hz}\right), 7.76-7.87(\mathrm{~m}, 2 \mathrm{H})$, 7.99-8.02 (m, 1H), 8.13-8.16 (m, 1H); ${ }^{13} \mathrm{C}$ NMR (DMSO- $d_{6}, 63 \mathrm{MHz}$ ): $\delta=14.0,19.6,22.6,27.5,28.9,29.0,29.1,31.8,81.0,101.6,128.3,129.9$, 131.0, 133.1, 139.7, 142.4, 143.8, 146.7, 175.5; IR (ATR, $\left.\mathrm{cm}^{-1}\right): \nu=3302$ (w), 3066 (w), 2956 (w), 2918 (s), 2852 (m), $2284(\mathrm{w}), 2205$ (m), 1661 (s), 1607 (w), $1574(w), 1553(w), 1531$ (m), $1504(w), 1479(w)$, 1466 (m), 1458 (m), 1427 (w), 1415 (w), $1380(\mathrm{w}), 1349$ (m), 1324 (w), 1315 (w), 1290 (m), 1279 (m), 1243 (w), $1230(\mathrm{w}), 1200(\mathrm{w})$, $1162(\mathrm{~s}), 1129(\mathrm{~m}), 1118(\mathrm{~m}), 1108(\mathrm{~m}), 1062(\mathrm{w}), 1045(\mathrm{~s}), 1028(\mathrm{~m})$; $\mathrm{UV}\left(\mathrm{CH}_{2} \mathrm{Cl}_{2}, \mathrm{~nm}\right): \lambda_{\max }(\log \varepsilon)=238$ (5.27), 248 (5.29), 310 (4.93); MS (EI, $70 \mathrm{eV}): m / z(\%)=328\left(\mathrm{M}^{+}, 85\right), 243(71), 191(44), 163(100), 129$ (14), 102 (19), 65 (9), 41 (8); HRMS (ESI): calcd for $\mathrm{C}_{19} \mathrm{H}_{22} \mathrm{ClN}_{2} \mathrm{O}$ $\left([\mathrm{M}+\mathrm{H}]^{+},{ }^{35} \mathrm{Cl}\right) \quad 329.14152$, found 329.14231, $\left([\mathrm{M}+\mathrm{Na}]^{+},{ }^{37} \mathrm{Cl}\right)$ 353.12115, found 353.12139. Anal. Calcd for $\mathrm{C}_{19} \mathrm{H}_{21} \mathrm{ClN}_{2} \mathrm{O}$ (328.84): C, 69.40; H, 6.44; N, 8.52. Found: C, 69.62; H, 6.71; N, 8.89.

\subsection{1-Cyclohexyl-2-phenylpyrido[2,3-b]quinoxalin-4(1H)- one (6a)}

Yellow solid, mp 238-240 ${ }^{\circ} \mathrm{C} .{ }^{1} \mathrm{H}$ NMR $\left(\mathrm{CDCl}_{3}, 300 \mathrm{MHz}\right)$ : $\delta=0.91-1.78(\mathrm{~m}, 8 \mathrm{H}), 2.97-3.11(\mathrm{~m}, 2 \mathrm{H}), 3.99-4.09(\mathrm{~m}, 1 \mathrm{H}), 6.35(\mathrm{~s}$, $1 \mathrm{H}), 7.37-7.49(\mathrm{~m}, 5 \mathrm{H}), 7.70-7.85(\mathrm{~m}, 2 \mathrm{H}), 8.00-8.03(\mathrm{~m}, 1 \mathrm{H})$, 8.37-8.40 (m, $1 \mathrm{H}) ;{ }^{13} \mathrm{C}$ NMR $\left(\mathrm{CDCl}_{3}, 63 \mathrm{MHz}\right): \delta=25.3,26.5,30.6$, $64.3,114.0,127.4,127.8,128.9,129.5,129.8,130.8,132.6,136.3,137.6$, 140.5, 141.8, 146.5, 157.8, 177.8; IR (ATR, $\left.\mathrm{cm}^{-1}\right)$ : $\nu=3057(\mathrm{w}), 3026$ (w), 2992 (w), $2950(\mathrm{w}), 2928$ (w), $2914(\mathrm{~m}), 2854(\mathrm{~m}), 1631$ (s), 1578 (m), 1545 (s), 1538 (m), $1504(\mathrm{w}), 1489(\mathrm{~m}), 1476(\mathrm{~s}), 1453(\mathrm{~m})$, $1442(\mathrm{~m}), 1427(\mathrm{~s}), 1417(\mathrm{~m}), 1393(\mathrm{~m}), 1352(\mathrm{~s}), 1340(\mathrm{~s}), 1304(\mathrm{~m})$, $1268(\mathrm{~s}), 1242(\mathrm{~m}), 1230(\mathrm{~m}), 1192(\mathrm{w}), 1178(\mathrm{w}), 1165(\mathrm{w}), 1154(\mathrm{w})$, $1139(\mathrm{~m}), 1122$ (s), $1098(\mathrm{~m}), 1072(\mathrm{~m}), 1047(\mathrm{~m}), 1029(\mathrm{~m}), 1020(\mathrm{~m})$; $\mathrm{UV}\left(\mathrm{CH}_{2} \mathrm{Cl}_{2}, \mathrm{~nm}\right): \lambda_{\max }(\log \varepsilon)=237$ (5.37), 247 (5.38), 311 (5.01), 434 (4.09); MS (EI, $70 \mathrm{eV}): m / z(\%)=355\left(\mathrm{M}^{+}, 13\right), 298(2), 273(100), 245$ (45), 205 (5), 129 (5), 102 (11), 55 (6), 41 (6); HRMS (ESI): calcd for $\mathrm{C}_{23} \mathrm{H}_{22} \mathrm{~N}_{3} \mathrm{O}[\mathrm{M}+\mathrm{H}]^{+}$356.17574, found 356.17642. Anal. Calcd for $\mathrm{C}_{23} \mathrm{H}_{21} \mathrm{~N}_{3} \mathrm{O}$ (355.432): C, 77.72; H, 5.96; N, 11.82. Found: C, 77.79; H, 5.92; N, 11.82 .

\subsection{1-Heptyl-2-phenylpyrido[2,3-b]quinoxalin-4(1H)-one (6b)}

Yellow solid, mp $136-138{ }^{\circ} \mathrm{C} .{ }^{1} \mathrm{H}$ NMR $\left(\mathrm{CDCl}_{3}, 300 \mathrm{MHz}\right): \delta=0.77$ (t, $\left.3 \mathrm{H},{ }^{3} \mathrm{~J}=6.9 \mathrm{~Hz}\right), 1.09-1.18(\mathrm{~m}, 8 \mathrm{H}), 1.56-1.63(\mathrm{~m}, 2 \mathrm{H}), 4.30-4.35$ $(\mathrm{m}, 2 \mathrm{H}), 6.39(\mathrm{~s}, 1 \mathrm{H}), 7.40-7.49(\mathrm{~m}, 5 \mathrm{H}), 7.71-7.86(\mathrm{~m}, 2 \mathrm{H})$, 8.00-8.03 (m, 1H), 8.39-8.43 (m, $1 \mathrm{H}) ;{ }^{13} \mathrm{C} \mathrm{NMR}\left(\mathrm{CDCl}_{3}, 63 \mathrm{MHz}\right)$ : $\delta=14.0,22.4,26.4,28.4,28.8,31.4,46.8,113.8,128.0,128.1,128.8$, $129.4,129.8,130.9,132.7,135.2,137.2,140.9,142.7,145.5,156.8$, 177.8; IR (ATR, $\mathrm{cm}^{-1}$ ): $\nu=3055$ (w), 2999 (w), 2951 (m), 2923 (m), $2852(\mathrm{~m}), 1637$ (s), 1633 (s), $1570(\mathrm{~m}), 1544(\mathrm{~s}), 1489(\mathrm{~m}), 1475$ (s), $1455(\mathrm{~m}), 1443(\mathrm{~s}), 1427(\mathrm{~m}), 1403(\mathrm{~m}), 1380(\mathrm{w}), 1353(\mathrm{~s}), 1300(\mathrm{~m})$, $1286(\mathrm{~m}), 1273(\mathrm{~m}), 1260(\mathrm{~m}), 1236(\mathrm{~m}), 1223(\mathrm{~m}), 1181(\mathrm{w}), 1164$ (m), 1132 (m), 1085 (w), 1074 (w), 1037 (w), 1003 (w); UV $\left(\mathrm{CH}_{2} \mathrm{Cl}_{2}\right.$, $\mathrm{nm}): \lambda_{\max }(\log \varepsilon)=228$ (5.71), 250 (5.55), 295 (5.35), 432 (4.04); MS (EI, $70 \mathrm{eV}): m / z(\%)=371\left(\mathrm{M}^{+}, 52\right), 342(10), 314(13), 286(51), 273$ (100), 245 (42), 129 (25), 102 (15), 41 (8); HRMS (ESI): calcd for $\mathrm{C}_{24} \mathrm{H}_{26} \mathrm{~N}_{3} \mathrm{O}[\mathrm{M}+\mathrm{H}]^{+}$372.20704, found 372.20713. Anal. Calcd for $\mathrm{C}_{24} \mathrm{H}_{25} \mathrm{~N}_{3} \mathrm{O}$ (371.475): C, 77.60; H, 6.78; N, 11.31. Found: C, 77.73; H, $6.79 ; \mathrm{N}, 11.42$.

\subsection{1-(2-Hydroxyethyl)-2-phenylpyrido[2,3-b]quinoxalin- $4(1 H)$-one (6c)}

Yellow solid, $\mathrm{mp} 243-245^{\circ} \mathrm{C} .{ }^{1} \mathrm{H} \mathrm{NMR}\left(\mathrm{CDCl}_{3}, 300 \mathrm{MHz}\right): \delta=4.04$ $\left(\mathrm{t},{ }^{3} \mathrm{~J}=4.9 \mathrm{~Hz}, 2 \mathrm{H}\right), 4.57\left(\mathrm{t}, 2 \mathrm{H},{ }^{3} \mathrm{~J}=4.9 \mathrm{~Hz}\right), 6.21(\mathrm{~s}, 1 \mathrm{H}), 7.46-7.72(\mathrm{~m}$, $7 \mathrm{H}), 7.86-7.89(\mathrm{~m}, 1 \mathrm{H}), 8.04-8.07(\mathrm{~m}, 1 \mathrm{H}) ;{ }^{13} \mathrm{C} \mathrm{NMR}\left(\mathrm{CDCl}_{3}\right.$, $63 \mathrm{MHz}): \delta=48.3,58.0,113.0,127.7,128.5,129.5,129.6,130.0,133.1$, 135.2, 137.2, 139.8, 141.5, 145.7, 157.0, 176.3; IR (ATR, $\left.\mathrm{cm}^{-1}\right): \nu=3335$ (w), 3050 (w), 2955 (w), 2924 (w), 2877 (w), 2730 (w), 1622 (s), $1615(\mathrm{~s}), 1581(\mathrm{w}), 1565(\mathrm{w}), 1546(\mathrm{~m}), 1538(\mathrm{~m}), 1494(\mathrm{~m}), 1474(\mathrm{~s})$ $1449(\mathrm{~m}), 1427(\mathrm{~m}), 1405(\mathrm{~m}), 1377(\mathrm{w}), 1353(\mathrm{~s}), 1338(\mathrm{~m}), 1295(\mathrm{w})$, $1282(\mathrm{~m}), 1262(\mathrm{~m}), 1230(\mathrm{~m}), 1188(\mathrm{w}), 1168(\mathrm{w}), 1147(\mathrm{~m}), 1116(\mathrm{w})$, $1071(\mathrm{~m}), 1053(\mathrm{~m}), 1010(\mathrm{w}) ; \mathrm{UV}\left(\mathrm{CH}_{2} \mathrm{Cl}_{2}, \mathrm{~nm}\right): \lambda_{\max }(\log \varepsilon)=228$ (5.71), 247 (5.55), 295 (5.33), 431 (3.99); MS (EI, $70 \mathrm{eV}): m / z(\%)=$ $317\left(\mathrm{M}^{+}, 22\right), 298$ (11), 286 (74), 273 (100), 257 (13), 245 (33), 207 (14), 129 (32), 102 (17); HRMS (ESI): calcd for $\mathrm{C}_{19} \mathrm{H}_{16} \mathrm{~N}_{3} \mathrm{O}_{2}[\mathrm{M}+\mathrm{H}]^{+}$ 318.12370, found 318.12453 .

\subsection{1-Benzyl-2-phenylpyrido[2,3-b]quinoxalin-4(1H)-one (6d)}

Pale orange solid, mp 205-208 ${ }^{\circ} \mathrm{C} .{ }^{1} \mathrm{H}$ NMR $\left(\mathrm{CDCl}_{3}, 300 \mathrm{MHz}\right)$ : $\delta=5.65(\mathrm{~s}, 2 \mathrm{H}), 6.44(\mathrm{~s}, 1 \mathrm{H}), 6.79-6.82(\mathrm{~m}, 2 \mathrm{H}), 7.08-7.09(\mathrm{~m}, 3 \mathrm{H})$ $7.20-7.41(\mathrm{~m}, 5 \mathrm{H}), 7.68-7.78(\mathrm{~m}, 2 \mathrm{H}), 7.89-7.93(\mathrm{~m}, 1 \mathrm{H}), 8.37-8.41$ $(\mathrm{m}, 1 \mathrm{H}) ;{ }^{13} \mathrm{C} \mathrm{NMR}\left(\mathrm{CDCl}_{3}, 63 \mathrm{MHz}\right): \delta=49.6,114.2,126.6,127.4,128.0$, 128.3, 128.5, 128.6, 129.6, 129.9, 130.9, 132.9, 134.8, 137.0, 137.0, 141.1, 142.7, 145.8, 157.1, 177.9; IR (ATR, $\left.\mathrm{cm}^{-1}\right): \nu=3110$ (w), 3058 (w), 3044 (w), 3023 (w), 2999 (w), 2960 (w), 1641 (s), 1622 (m), 1574 (m), $1548(\mathrm{~m}), 1495(\mathrm{w}), 1485(\mathrm{w}), 1476(\mathrm{~s}), 1453(\mathrm{~m}), 1444(\mathrm{~s}), 1410$ (m), $1401(\mathrm{~m}), 1359(\mathrm{~s}), 1336(\mathrm{~m}), 1330(\mathrm{~m}), 1293(\mathrm{w}), 1275(\mathrm{~s}), 1253$ (m), 1231 (m), 1198 (w), $1188(\mathrm{w}), 1159(\mathrm{w}), 1138(\mathrm{~m}), 1103(\mathrm{w}), 1078$ (w), 1039 (w), 1023 (w); UV $\left(\mathrm{CH}_{2} \mathrm{Cl}_{2}, \mathrm{~nm}\right): \lambda_{\max }(\log \varepsilon)=228$ (5.79), 254 (5.64), 296 (5.45), 428 (4.20); MS (EI, $70 \mathrm{eV}): m / z(\%)=363\left(\mathrm{M}^{+}\right.$, 57), 334 (3), 273 (15), 245 (12), 129 (5), 91 (100), 69 (13), 57 (12), 44 (44); HRMS (ESI): calcd for $\mathrm{C}_{24} \mathrm{H}_{18} \mathrm{~N}_{3} \mathrm{O}[\mathrm{M}+\mathrm{H}]^{+} 364.14444$, found 364.14515. Anal. Calcd for $\mathrm{C}_{24} \mathrm{H}_{17} \mathrm{~N}_{3} \mathrm{O}$ (363.411): C, 79.32; $\mathrm{H}, 4.72 ; \mathrm{N}$, 11.56. Found: C, 79.34; H, 4.75; N, 11.57 .

\subsection{1-Phenethyl-2-phenylpyrido[2,3-b]quinoxalin-4(1H)- one (6e)}

Yellow solid, $\mathrm{mp} 295-297^{\circ} \mathrm{C} .{ }^{1} \mathrm{H} \mathrm{NMR}\left(\mathrm{CDCl}_{3}, 300 \mathrm{MHz}\right): \delta=2.92$ $\left(\mathrm{t}, 2 \mathrm{H},{ }^{3} \mathrm{~J}=7.6 \mathrm{~Hz}\right), 4.55\left(\mathrm{t}, 2 \mathrm{H},{ }^{3} \mathrm{~J}=7.6 \mathrm{~Hz}\right), 6.36(\mathrm{~s}, 1 \mathrm{H}), 6.79-6.83(\mathrm{~m}$, 2H), 7.11-7.14 (m, 3H), 7.25-7.29 (m, 2H), 7.45-7.48 (m, 3H), $7.73-7.90(\mathrm{~m}, 2 \mathrm{H}), 8.05-8.09(\mathrm{~m}, 1 \mathrm{H}), 8.41-8.44(\mathrm{~m}, 1 \mathrm{H}) ;{ }^{13} \mathrm{C} \mathrm{NMR}$ $\left(\mathrm{CDCl}_{3}, 63 \mathrm{MHz}\right): \delta=35.2,48.3,113.8,126.7,128.0,128.1,128.6,128.7$, 128.8, 129.6, 129.9, 131.0, 132.9, 135.0, 137.2, 137.7, 140.9, 142.7, 145.5, 156.8, 177.8; IR (ATR, cm $\left.{ }^{-1}\right): \nu=3059(\mathrm{w}), 3021(\mathrm{w}), 2992(\mathrm{w})$, $2953(\mathrm{w}), 1651$ (w), $1629(\mathrm{~s}), 1579(\mathrm{~m}), 1574(\mathrm{~m}), 1551(\mathrm{~m}), 1545(\mathrm{~m})$, 1495 (m), 1475 (m), 1448 (m), 1441 (m), 1425 (m), 1407 (m), 1373 
(m), $1254(\mathrm{~m}), 1333(\mathrm{~m}), 1300(\mathrm{w}), 1288(\mathrm{~m}), 1269(\mathrm{~m}), 1258(\mathrm{~m})$, $1236(\mathrm{~m}), 1226(\mathrm{~m}), 1216(\mathrm{~m}), 1176(\mathrm{w}), 1164(\mathrm{~m}), 1142(\mathrm{~m}), 1105(\mathrm{w})$, 1075 (w), 1039 (w), 1028 (w), 1001 (m); UV ( $\left.\mathrm{CH}_{2} \mathrm{Cl}_{2}, \mathrm{~nm}\right): \lambda_{\max }$ $(\log \varepsilon)=228$ (5.72), 247 (5.59), 295 (5.39), 428 (4.22); MS (EI, $70 \mathrm{eV):}$ $m / z(\%)=377\left(\mathrm{M}^{+}, 9\right), 286$ (77), 273 (100), 245 (14), 129 (26), 102 (10), 69 (9), 44 (2); HRMS (ESI): calcd for $\mathrm{C}_{25} \mathrm{H}_{20} \mathrm{~N}_{3} \mathrm{O}[\mathrm{M}+\mathrm{H}]^{+}$ 378.16009 , found 378.15962 .

\subsection{1-(2-Methoxyphenethyl)-2-phenylpyrido[2,3-b]quinox- alin-4(1H)-one (6f)}

Yellow solid, $\mathrm{mp} 200-202{ }^{\circ} \mathrm{C} .{ }^{1} \mathrm{H}$ NMR $\left(\mathrm{CDCl}_{3}, 300 \mathrm{MHz}\right): \delta=2.90$ $\left(\mathrm{t}, 2 \mathrm{H},{ }^{3} \mathrm{~J}=6.7 \mathrm{~Hz}\right), 3.32(\mathrm{~s}, 3 \mathrm{H}), 4.64\left(\mathrm{t}, 2 \mathrm{H},{ }^{3} \mathrm{~J}=6.6 \mathrm{~Hz}\right), 6.25(\mathrm{~s}, 1 \mathrm{H})$, 6.55-6.58 (m, 1H), 6.66-6.67 (m, 2H), 7.02-7.05 (m, 3H), 7.31-7.41 $(\mathrm{m}, 3 \mathrm{H}), 7.72-7.87(\mathrm{~m}, 1 \mathrm{H}), 8.03-8.06(\mathrm{~m}, 1 \mathrm{H}), 8.40-8.43(\mathrm{~m}, 2 \mathrm{H})$; ${ }^{13} \mathrm{C}$ NMR $\left(\mathrm{CDCl}_{3}, 63 \mathrm{MHz}\right): \delta=29.6,47.0,54.7,110.0,113.7,120.5$, $126.1,128.0,128.1,128.2,128.5,129.4,129.4,130.7,130.9,132.7$, 135.2, 137.4, 140.8, 142.6, 145.8, 157.3, 157.5, 177.8; IR (ATR, $\left.\mathrm{cm}^{-1}\right)$ : $\nu=3055$ (w), 3019 (w), 2938 (w), $2834(\mathrm{w}), 1627$ (s), 1601 (w), 1579 (m), $1545(\mathrm{~m}), 1492(\mathrm{~m}), 1473(\mathrm{~s}), 1462(\mathrm{~m}), 1451(\mathrm{~m}), 1441(\mathrm{~m}), 1423$ (m), $1399(\mathrm{~m}), 1370(\mathrm{w}), 1353(\mathrm{~s}), 1334(\mathrm{~m}), 1290(\mathrm{~m}), 1268(\mathrm{~m}), 1244$ (s), $1215(\mathrm{~m}), 1175(\mathrm{w}), 1160(\mathrm{~m}), 1139(\mathrm{~m}), 1093(\mathrm{~m}), 1074(\mathrm{w}), 1039$ (m), 1030 (m), $1001(\mathrm{~m}) ; \mathrm{UV}\left(\mathrm{CH}_{2} \mathrm{Cl}_{2}, \mathrm{~nm}\right): \lambda_{\max }(\log \varepsilon)=228$ (5.70), 247 (5.56), 295 (5.36), 432 (4.04); MS (EI, $70 \mathrm{eV}): m / z(\%)=407\left(\mathrm{M}^{+}\right.$, 44), 286 (52), 273 (100), 245 (21), 129 (32), 91 (15), 77 (6); HRMS (ESI): calcd for $\mathrm{C}_{26} \mathrm{H}_{22} \mathrm{~N}_{3} \mathrm{O}_{2}[\mathrm{M}+\mathrm{H}]^{+} 408.17065$, found 408.17072 . Anal. Calcd for $\mathrm{C}_{26} \mathrm{H}_{21} \mathrm{~N}_{3} \mathrm{O}_{2}$ (407.464): C, 76.64; H, 5.19; N, 10.13 . Found: C, 76.77; H, 5.31; N, 10.31 .

\subsection{1,2-Diphenylpyrido[2,3-b]quinoxalin-4(1H)-one $(6 g)$}

Yellow crystals, mp 305-307 ${ }^{\circ} \mathrm{C} .{ }^{1} \mathrm{H}$ NMR $\left(\mathrm{CDCl}_{3}, 300 \mathrm{MHz}\right)$ : $\delta=6.63(\mathrm{~s}, 1 \mathrm{H}), 7.13-7.18(\mathrm{~m}, 6 \mathrm{H}), 7.27-7.32(\mathrm{~m}, 4 \mathrm{H}), 7.68-7.78(\mathrm{~m}$, $3 \mathrm{H}), 8.38-8.41(\mathrm{~m}, 1 \mathrm{H}) ;{ }^{13} \mathrm{C} \mathrm{NMR}\left(\mathrm{CDCl}_{3}, 63 \mathrm{MHz}\right): \delta=112.9,127.1$, $127.3,127.4,127.8,128.0,128.2,128.6,129.2,129.8,131.7,134.2$, $135.4,137.3,140.1,141.7,145.9,155.5,177.2$; IR (ATR, $\left.\mathrm{cm}^{-1}\right): \nu=3054$ (w), $3043(\mathrm{w}), 1634(\mathrm{~s}), 1595(\mathrm{w}), 1572(\mathrm{w}), 1550(\mathrm{~m}), 1540(\mathrm{~m}), 1496$ (m), 1476 (s), 1455 (w), 1443 (w), $1421(\mathrm{w}), 1401(\mathrm{~m}), 1358(\mathrm{~m}), 1337$ (w), $1316(\mathrm{w}), 1299(\mathrm{w}), 1273(\mathrm{~m}), 1250(\mathrm{w}), 1231(\mathrm{w}), 1211(\mathrm{w}), 1188$ (w), $1164(w), 1148$ (w), 1137 (w), $1112(w), 1075$ (w), 1033 (w), 1003 (w); UV $\left(\mathrm{CH}_{2} \mathrm{Cl}_{2}, \mathrm{~nm}\right): \lambda_{\max }(\log \varepsilon)=236$ (5.37), 248 (5.37), 311 (5.00), 428 (4.08); MS (EI, $70 \mathrm{eV}): m / z(\%)=349\left(\mathrm{M}^{+}, 95\right), 320(30)$, 218 (10), 129 (6), 102 (4), 77 (7); HRMS (ESI): calcd for $\mathrm{C}_{23} \mathrm{H}_{16} \mathrm{~N}_{3} \mathrm{O}$ $[\mathrm{M}+\mathrm{H}]^{+} 350.12879$, found 350.12812. Anal. Calcd for $\mathrm{C}_{23} \mathrm{H}_{15} \mathrm{~N}_{3} \mathrm{O}$ (349.385): C, 79.07; H, 4.33; N, 12.03. Found: C, 79.16; H, 4.41; N, 12.05 .

\subsection{2-Phenyl-1-(3,4,5-trimethoxyphenyl)pyrido[2,3-b]qui- noxalin-4(1H)-one $(6 \mathrm{~h})$}

Yellow solid, $\mathrm{mp} 281-283{ }^{\circ} \mathrm{C} .{ }^{1} \mathrm{H}$ NMR $\left(\mathrm{CDCl}_{3}, 300 \mathrm{MHz}\right): \delta=3.63$ (s, 6H), $3.79(\mathrm{~s}, 3 \mathrm{H}), 6.38(\mathrm{~s}, 2 \mathrm{H}), 6.59(\mathrm{~s}, 1 \mathrm{H}), 7.20-7.21(\mathrm{~s}, 5 \mathrm{H})$, 7.69-7.85 (m, 3H), 8.36-8.40 (m, 1H); ${ }^{13} \mathrm{C} \mathrm{NMR}\left(\mathrm{CDCl}_{3}, 63 \mathrm{MHz}\right)$ : $\delta=56.3,61.0,108.2,113.9,128.1,128.3,128.6,129.3,129.7,130.8$, 132.7, 133.5, 135.4, 136.3, 138.0, 141.1, 142.7, 146.8, 153.0, 156.4, 178.2; IR (ATR, $\mathrm{cm}^{-1}$ ): $\nu=3060$ (w), 3011 (w), 2985 (w), 2967 (w), 2935 (w), 2841 (w), 2823 (w), 1636 (m), 1598 (m), 1574 (m), 1552 (m), 1538 (w), $1495(\mathrm{~m}), 1475(\mathrm{~m}), 1464(\mathrm{~m}), 1453(\mathrm{~m}), 1416(\mathrm{~m})$, 1398 (m), 1365 (m), 1335 (m), 1308 (m), 1288 (w), 1265 (m), 1232 (s), $1168(\mathrm{w}), 1149$ (m), 1108 (s), 1098 (s), 1038 (w), 1030 (w), 1004 (s); UV $\left(\mathrm{CH}_{2} \mathrm{Cl}_{2}, \mathrm{~nm}\right): \lambda_{\max }(\log \varepsilon)=229$ (5.71), 254 (5.57), 295 (5.37), 431 (4.07); MS (EI, $70 \mathrm{eV}): m / z(\%)=439\left(\mathrm{M}^{+}, 100\right), 424$ (30), 392 (10), 251 (7), 141 (5), 102 (5); HRMS (ESI): calcd for $\mathrm{C}_{26} \mathrm{H}_{22} \mathrm{~N}_{3} \mathrm{O}_{4}$ $[\mathrm{M}+\mathrm{H}]^{+} 440.16048$, found 440.16076. Anal. Calcd for $\mathrm{C}_{26} \mathrm{H}_{21} \mathrm{~N}_{3} \mathrm{O}_{4}$ (439.463): C, 71.06; H, 4.82; N, 9.56. Found: C, 71.11; H, 4.75; N, 9.90.

\subsection{2-(4-tert-Butylphenyl)-1-cyclohexylpyrido[2,3-b]qui- noxalin-4(1H)-one $(6 \mathbf{i})$}

Yellow solid, mp 254-256 ${ }^{\circ} \mathrm{C} .{ }^{1} \mathrm{H}$ NMR $\left(\mathrm{CDCl}_{3}, 300 \mathrm{MHz}\right)$ : $\delta=0.94-1.03(\mathrm{~m}, 2 \mathrm{H}), 1.18-1.26(\mathrm{~m}, 1 \mathrm{H}), 1.32(\mathrm{~s}, 9 \mathrm{H}), 1.53-1.58(\mathrm{~m}$, $1 \mathrm{H}), 1.71-1.78(\mathrm{~m}, 4 \mathrm{H}), 2.96-3.10(\mathrm{~m}, 2 \mathrm{H}), 4.03-4.13(\mathrm{~m}, 1 \mathrm{H}), 6.35$ (s, $1 \mathrm{H}), 7.29-7.32(\mathrm{~m}, 2 \mathrm{H}), 7.45-7.48(\mathrm{~m}, 2 \mathrm{H}), 7.68-7.73(\mathrm{~m}, 1 \mathrm{H})$, 7.77-7.83 (m, 1H), 7.99-8.02 (m, $1 \mathrm{H}), 8.35-8.38(\mathrm{~m}, 1 \mathrm{H}) ;{ }^{13} \mathrm{C}$ NMR $\left(\mathrm{CDCl}_{3}, 63 \mathrm{MHz}\right): \delta=25.3,26.6,30.6,31.2,34.9,64.2,114.1$, $125.8,127.3,127.8,129.4,130.8,132.5,133.3,137.6,140.4,141.8$, 146.5, 153.2, 158.2, 177.8; IR (ATR, $\left.\mathrm{cm}^{-1}\right): \nu=3060$ (w), 3045 (w), 2961 (m), $2929(\mathrm{~m}), 2850$ (m), 1643 (s), 1615 (w), 1577 (m), 1548 (m), 1520 (w), 1505 (m), $1488(\mathrm{~m}), 1475$ (m), $1454(\mathrm{~m}), 1424(\mathrm{~m})$, 1412 (w), 1397 (m), 1353 (s), 1335 (m), 1301 (m), 1267 (s), 1247 (m), 1232 (m), 1201 (w), 1165 (w), $1154(\mathrm{w}), 1134(\mathrm{~m}), 1121(\mathrm{~m})$, 1094 (m), 1047 (m), 1020 (m), 1003 (w); UV ( $\left.\mathrm{CH}_{2} \mathrm{Cl}_{2}, \mathrm{~nm}\right): \lambda_{\max }$ $(\log \varepsilon)=228$ (5.69), 248 (5.57), 291 (5.37), 426 (4.19); MS (EI, $70 \mathrm{eV}): \mathrm{m} / z(\%)=411\left(\mathrm{M}^{+}, 10\right), 329$ (100), 314 (44), 286 (22), 272 (11), 129 (9), 55 (7), 41 (8); HRMS (ESI): calcd for $\mathrm{C}_{27} \mathrm{H}_{30} \mathrm{~N}_{3} \mathrm{O}$ $[\mathrm{M}+\mathrm{H}]^{+}$412.23834, found 412.23900. Anal. Calcd for $\mathrm{C}_{27} \mathrm{H}_{29} \mathrm{~N}_{3} \mathrm{O}$ (411.539): C, 78.80; H, 7.10; N, 10.21. Found: C, 78.39; H, 7.19; N, 10.52 .

\subsection{2-(4-tert-Butylphenyl)-1-heptylpyrido[2,3-b]quinox- alin-4(1H)-one $(6 \mathbf{j})$}

Yellow solid, mp $167-169{ }^{\circ} \mathrm{C} .{ }^{1} \mathrm{H}$ NMR $\left(\mathrm{CDCl}_{3}, 300 \mathrm{MHz}\right): \delta=0.76$ $\left(\mathrm{t}, 3 \mathrm{H},{ }^{3} \mathrm{~J}=6.8 \mathrm{~Hz}\right), 1.07-1.12(\mathrm{~m}, 8 \mathrm{H}), 1.33(\mathrm{~s}, 9 \mathrm{H}), 1.58-1.63(\mathrm{~m}, 2 \mathrm{H})$, $4.34\left(\mathrm{t}, 2 \mathrm{H},{ }^{3} \mathrm{~J}=7.6 \mathrm{~Hz}\right), 6.40(\mathrm{~s}, 1 \mathrm{H}), 7.32-7.49(\mathrm{~m}, 4 \mathrm{H}), 7.71-7.85(\mathrm{~m}$, $2 \mathrm{H}), 7.99-8.02(\mathrm{~m}, 1 \mathrm{H}), 8.39-8.42(\mathrm{~m}, 1 \mathrm{H}) ;{ }^{13} \mathrm{C} \mathrm{NMR}\left(\mathrm{CDCl}_{3}\right.$, $63 \mathrm{MHz}$ ): $\delta=13.9,22.4,26.3,28.3,28.7,31.2,31.4,34.8,46.7,113.8$, 125.6, 127.8, 127.9, 129.3, 130.9, 132.2, 132.6, 137.2, 140.8, 142.7, 145.5, 153.1, 157.1, 177.7; IR (ATR, $\left.\mathrm{cm}^{-1}\right): \nu=3062$ (w), 3046 (w), 3011 (w), $2957(\mathrm{~m}), 2923(\mathrm{~m}), 2912(\mathrm{~m}), 2859(\mathrm{~m}), 1637(\mathrm{~s}), 1573(\mathrm{~m})$, 1547 (s), 1510 (m), 1489 (s), 1476 (s), 1465 (s), 1451 (s), 1431 (m), $1404(\mathrm{~m}), 1381(\mathrm{w}), 1360(\mathrm{~s}), 1352(\mathrm{~s}), 1335(\mathrm{~m}), 1301(\mathrm{w}), 1285(\mathrm{~m})$, 1266 (s), 1255 (s), $1230(\mathrm{~m}), 1216(\mathrm{w}), 1201(\mathrm{~m}), 1161(\mathrm{~m}), 1145(\mathrm{w})$, 1134 (m), 1125 (m), 1110 (w), 1084 (w), 1028 (w), 1003 (w); UV $\left(\mathrm{CH}_{2} \mathrm{Cl}_{2}, \mathrm{~nm}\right): \lambda_{\max }(\log \varepsilon)=228$ (5.72), 247 (5.59), 293 (5.39), 428 (4.19); MS (EI, $70 \mathrm{eV}): m / z(\%)=427\left(\mathrm{M}^{+}, 60\right), 398$ (9), 370 (16), 342 (20), 329 (100), 314 (31), 286 (36), 272 (10), 129 (18), 57 (6), 41 (4); HRMS (ESI): calcd for $\mathrm{C}_{28} \mathrm{H}_{34} \mathrm{~N}_{3} \mathrm{O}[\mathrm{M}+\mathrm{H}]^{+} 428.26964$, found 428.26997.

\subsection{2-(4-tert-Butylphenyl)-1-phenethylpyrido[2,3-b]quinox- alin-4(1H)-one (6k)}

Yellow solid, $\mathrm{mp} 281-283^{\circ} \mathrm{C} .{ }^{1} \mathrm{H}$ NMR $\left(\mathrm{CDCl}_{3}, 300 \mathrm{MHz}\right): \delta=1.36$ (s, 9H), 2.89 (t, 2H, $\left.{ }^{3} \mathrm{~J}=7.7 \mathrm{~Hz}\right), 4.57\left(\mathrm{t}, 2 \mathrm{H},{ }^{3} \mathrm{~J}=7.7 \mathrm{~Hz}\right), 6.39(\mathrm{~s}, 1 \mathrm{H})$, 6.77-6.80 (m, 2H), 7.10-7.12 (m, 3H), 7.23-7.25 (m, 2H), 7.46-7.49 $(\mathrm{m}, 2 \mathrm{H}), 7.73-7.89(\mathrm{~m}, 2 \mathrm{H}), 8.05-8.08(\mathrm{~m}, 1 \mathrm{H}), 8.41-8.44(\mathrm{~m}, 1 \mathrm{H})$; ${ }^{13} \mathrm{C}$ NMR $\left(\mathrm{CDCl}_{3}, 63 \mathrm{MHz}\right): \delta=31.3,34.9,35.2,48.1,113.8,125.7$, 126.7, 127.9, 127.9, 128.5, 128.7, 129.5, 130.9, 132.0, 132.8, 137.2, $137.8,140.8,142.7,145.5,153.2,156.9,177.8 ;$ IR $\left(\right.$ ATR, $\left.\mathrm{cm}^{-1}\right): \nu=3054$ (w), 3021 (w), 2952 (w), 2901 (w), $2867(w), 1632(\mathrm{~s}), 1579(\mathrm{~m})$, 1546 (m), 1505 (w), 1490 (m), 1475 (m), 1463 (m), 1448 (m), 1424 (m), $1404(\mathrm{~m}), 1373(\mathrm{w}), 1354(\mathrm{~m}), 1332(\mathrm{~m}), 1287(\mathrm{~m}), 1267(\mathrm{~m})$, $1234(\mathrm{~m}), 1213(\mathrm{~m}), 1201(\mathrm{w}), 1191(\mathrm{w}), 1178(\mathrm{w}), 1160(\mathrm{~m}), 1136(\mathrm{~m})$, 1119 (w), 1101 (w), 1073 (w), 1027 (w), 1015 (w); UV ( $\left.\mathrm{CH}_{2} \mathrm{Cl}_{2}, \mathrm{~nm}\right)$ : $\lambda_{\max }(\log \varepsilon)=228$ (5.67), 247 (5.53), 293 (5.33), 434 (4.04); MS (EI, $70 \mathrm{eV}): m / z(\%)=433\left(\mathrm{M}^{+}, 10\right), 329(100), 314(17), 286(87), 272(6)$, 129 (28), 102 (8), 57 (22), 41 (5); HRMS (ESI): calcd for $\mathrm{C}_{29} \mathrm{H}_{28} \mathrm{~N}_{3} \mathrm{O}$ $[\mathrm{M}+\mathrm{H}]^{+} 434.22269$, found 434.22380. Anal. Calcd for $\mathrm{C}_{29} \mathrm{H}_{27} \mathrm{~N}_{3} \mathrm{O}$ (433.544): C, 80.34; H, 6.28; N, 9.69. Found: C, 80.29; H, 6.61; N, 9.63 . 


\subsection{1-Cyclohexyl-2-octylpyrido[2,3-b]quinoxalin-4(1H)-one} (61)

Yellow solid, mp $115-117{ }^{\circ} \mathrm{C} .{ }^{1} \mathrm{H}$ NMR $\left(\mathrm{CDCl}_{3}, 300 \mathrm{MHz}\right)$ : $\delta=0.69-0.73(\mathrm{~m}, 3 \mathrm{H}), 1.12-1.30(\mathrm{~m}, 7 \mathrm{H}), 1.55-1.67(\mathrm{~m}, 5 \mathrm{H})$, $1.83-1.86(\mathrm{~m}, 2 \mathrm{H}), 2.45-2.46(\mathrm{~m}, 6 \mathrm{H}), 2.61-2.66(\mathrm{~m}, 2 \mathrm{H}), 3.07-3.10$ $(\mathrm{m}, 2 \mathrm{H}), 4.13-4.18(\mathrm{~m}, 1 \mathrm{H}), 6.23(\mathrm{~s}, 1 \mathrm{H}), 7.58-7.63(\mathrm{~m}, 1 \mathrm{H})$, 7.68-7.73 (m, 1H), 7.88-7.91 (m, 1H), 8.19-8.22 (m, 1H); ${ }^{13} \mathrm{C}$ NMR $\left(\mathrm{CDCl}_{3}, 63 \mathrm{MHz}\right): \delta=13.7,22.2,25.1,26.6,28.6,28.7,28.8,28.9,30.2$, 31.3, 35.4, 61.4, 112.3, 127.5, 129.1, 130.2, 132.1, 136.8, 139.8, 141.1, 146.0, 158.0, 177.6; IR (ATR, $\mathrm{cm}^{-1}$ ): $\nu=3058(\mathrm{w}), 3046(\mathrm{w}), 2923(\mathrm{~m})$, 2851 (w), $1639(\mathrm{~s}), 1576(\mathrm{w}), 1549(\mathrm{~m}), 1543(\mathrm{~m}), 1476(\mathrm{~m}), 1466(\mathrm{~m})$, 1423 (w), 1391 (w), 1348 (m), 1321 (w), 1295 (m), 1278 (m), 1230 (m), 1188 (w), 1159 (w), 1139 (w), 1118 (w), 1070 (w), 1053 (w), 1030 (w), $1017(w), 1002(w), 893(w), 870(w), 833(s), 775$ (s), $765(s)$, $724(\mathrm{~m}), 685$ (w), 677 (w), 661 (w), $610(\mathrm{w}) ; \mathrm{UV}\left(\mathrm{CH}_{2} \mathrm{Cl}_{2}, \mathrm{~nm}\right): \lambda_{\max }$ $(\log \varepsilon)=237$ (5.37), 248 (5.37), 310 (5.02), 421 (3.98); MS (EI, $70 \mathrm{eV})$ : $\mathrm{m} / \mathrm{z}(\%)=391\left(\mathrm{M}^{+}, 66\right), 310$ (23), 281 (29), 224 (52), 211 (100), 183 (22), 129 (4); HRMS (ESI): calcd for $\mathrm{C}_{25} \mathrm{H}_{34} \mathrm{~N}_{3} \mathrm{O}[\mathrm{M}+\mathrm{H}]^{+} 392.26964$, found 392.27078.

\subsection{2-Octyl-1-phenethylpyrido[2,3-b]quinoxalin-4(1H)-one (6m)}

Yellow solid, mp $144-146{ }^{\circ} \mathrm{C} .{ }^{1} \mathrm{H}$ NMR $\left(\mathrm{CDCl}_{3}, 300 \mathrm{MHz}\right)$ : $\delta=0.80-0.84(\mathrm{~m}, 3 \mathrm{H}), 1.22-1.35(\mathrm{~m}, 9 \mathrm{H}), 1.61-1.66(\mathrm{~m}, 3 \mathrm{H}), 2.56(\mathrm{t}$, $\left.2 \mathrm{H},{ }^{3} \mathrm{~J}=7.7 \mathrm{~Hz}\right), 3.08\left(\mathrm{t}, 2 \mathrm{H},{ }^{3} \mathrm{~J}=7.6 \mathrm{~Hz}\right), 4.70\left(\mathrm{t}, 2 \mathrm{H},{ }^{3} \mathrm{~J}=7.6 \mathrm{~Hz}\right), 6.35(\mathrm{~s}$, $1 \mathrm{H}), 7.17-7.27(\mathrm{~m}, 5 \mathrm{H}), 7.72-7.84(\mathrm{~m}, 1 \mathrm{H}), 7.81-7.84(\mathrm{~m}, 1 \mathrm{H})$, 8.03-8.06 (m, 1H), 8.37-8.40 (m, 1H); ${ }^{13} \mathrm{C} \mathrm{NMR}\left(\mathrm{CDCl}_{3}, 63 \mathrm{MHz}\right)$ : $\delta=14.0,22.6,28.5,29.1,29.2,29.3,31.7,33.7,35.4,46.2,111.7,127.0$, 128.0, 128.8, 128.8, 129.4, 130.9, 132.7, 137.0, 137.9, 140.7, 142.5, 145.4, 157.6, 177.9; IR (ATR, $\mathrm{cm}^{-1}$ ): $\nu=3048$ (w), 3024 (w), 2982 (w), 2948 (w), 2929 (m), 2856 (m), $1634(\mathrm{~s}), 1612(\mathrm{~m}), 1583(\mathrm{~m}), 1548$ (m), 1541 (m), $1489(\mathrm{~m}), 1475(\mathrm{~m}), 1466(\mathrm{~m}), 1435(\mathrm{~m}), 1418(\mathrm{~m})$, $1399(\mathrm{~m}), 1370(\mathrm{~m}), 1353(\mathrm{~s}), 1331(\mathrm{~m}), 1322(\mathrm{w}), 1299(\mathrm{~m}), 1292(\mathrm{~m})$, $1279(\mathrm{~m}), 1271(\mathrm{~m}), 1234(\mathrm{~m}), 1224(\mathrm{~m}), 1208(\mathrm{w}), 1169(\mathrm{~m}), 1156$ (m), $1134(\mathrm{~m}), 1121$ (m), $1092(\mathrm{w}), 1074(\mathrm{w}), 1030$ (m), 1013 (w); UV $\left(\mathrm{CH}_{2} \mathrm{Cl}_{2}, \mathrm{~nm}\right): \lambda_{\max }(\log \varepsilon)=228$ (5.73), 254 (5.60), 296 (5.40), 428 (4.21); MS (EI, $70 \mathrm{eV}): m / z(\%)=413\left(\mathrm{M}^{+}, 45\right), 322$ (100), 238 (16), 224 (30), 211 (73), 183 (14), 129 (13), 105 (9); HRMS (ESI): calcd for $\mathrm{C}_{27} \mathrm{H}_{32} \mathrm{~N}_{3} \mathrm{O}[\mathrm{M}+\mathrm{H}]^{+}$414.25399, found 414.25453. Anal. Calcd for $\mathrm{C}_{27} \mathrm{H}_{31} \mathrm{~N}_{3} \mathrm{O}$ (413.555): C, 78.42; H, 7.56; N, 10.16. Found: C, 78.39; H, 7.59; N, 10.22 .

\subsection{2-Octyl-1-(3,4,5-trimethoxyphenyl)pyrido[2,3-b]qui- noxalin-4(1H)-one $(6 n)$}

Yellow solid, mp $189-191{ }^{\circ} \mathrm{C} .{ }^{1} \mathrm{H}$ NMR $\left(\mathrm{CDCl}_{3}, 300 \mathrm{MHz}\right)$ : $\delta=0.77-0.81(\mathrm{~m}, 3 \mathrm{H}), 1.15-1.20(\mathrm{~m}, 10 \mathrm{H}), 1.52-1.59(\mathrm{~m}, 2 \mathrm{H}), 2.48(\mathrm{t}$, $\left.2 \mathrm{H},{ }^{3} \mathrm{~J}=7.7 \mathrm{~Hz}\right), 3.79(\mathrm{~s}, 6 \mathrm{H}), 3.94(\mathrm{~s}, 3 \mathrm{H}), 6.46(\mathrm{~s}, 2 \mathrm{H}), 6.47(\mathrm{~s}, 1 \mathrm{H})$, $7.64-7.79(\mathrm{~m}, 3 \mathrm{H}), 8.32-8.35(\mathrm{~m}, 1 \mathrm{H}) ;{ }^{13} \mathrm{C} \mathrm{NMR}\left(\mathrm{CDCl}_{3}, 63 \mathrm{MHz}\right)$ : $\delta=14.0,22.5,28.7,29.0,29.1,29.2,31.7,34.0,56.4,61.1,106.8,111.1$, 128.3, 129.4, 130.7, 132.4, 133.2, 136.2, 138.6, 140.9, 142.7, 147.2, 153.9, 158.3, 178.2; IR (ATR, $\mathrm{cm}^{-1}$ ): $\nu=3078(\mathrm{w}), 3061$ (w), $2922(\mathrm{~m})$, 2846 (w), 1640 (s), 1596 (m), 1581 (m), 1545 (m), 1500 (m), 1480 (m), 1467 (m), 1455, (m), 1419 (m), 1400 (m), $1361(\mathrm{~m}), 1341(\mathrm{~m})$, 1313 (m), 1274 (m), 1232 (s), 1206 (m), 1170 (w), 1144 (w), 1117 (s), $1070(\mathrm{~m}), 1024(\mathrm{w}), 1005(\mathrm{~m}) ; \mathrm{UV}\left(\mathrm{CH}_{2} \mathrm{Cl}_{2}, \mathrm{~nm}\right): \lambda(\log \varepsilon)=227(5.76)$, 254 (5.62), 296 (5.43), 428 (4.22); MS (EI): $m / z(\%)=475\left(\mathrm{M}^{+}, 60\right)$, 460 (8), 390 (48), 377 (100), 362 (19), 334 (10), 181 (8); HRMS (ESI): calcd for $\mathrm{C}_{28} \mathrm{H}_{34} \mathrm{~N}_{3} \mathrm{O}_{4}[\mathrm{M}+\mathrm{H}]^{+}$476.25438, found 476.25329. Anal. Calcd for $\mathrm{C}_{28} \mathrm{H}_{33} \mathrm{~N}_{3} \mathrm{O}_{4}$ (475.579): C, 70.71; $\mathrm{H}, 6.99 ; \mathrm{N}, 8.84$. Found: $\mathrm{C}$ 70.78; H, 6.93; N, 8.70.

\section{Acknowledgements}

Financial support by DAAD (scholarships for S.M. and A.G.) is gratefully acknowledged.

\section{References and notes}

1. Litvinov, V. P. Adv. Heterocycl. Chem. 2006, 91, 189-300.

2. (a) Matsumoto, J.; Miyamoto, T.; Minamida, A.; Nishimura, Y.; Egawa, H.; Nishimura, H. J. Med. Chem. 1984, 27, 292-301; (b) Miyamoto, T.; Egawa, H. Matsumoto, J. Chem. Pharm. Bull. 1987, 35, 2280-2285.

3. (a) Kaatz, G. W.; Seo, S. M.; Aeschlimann,J. R.; Houlihan, H. H.; Mercier, R.-C.; Rybak M. J. Antimicrob. Agents Chemother. 1998, 42, 254-256; (b) Vilsmaier, E.; Goerz, T Synthesis 1998, 739-744; (c) Garey, K. W.; Amsden, G. W. Pharmacotherapy 1999, 19, 21-34.

4. (a) Kotljarov, A.; Irgashev, R. A.; Iaroshenko, V. O.; Sevenard, D. V.; Sosnovskikh, V. Y. Synthesis 2009, 3233-3242; (b) Kotljarov, A.; Iaroshenko, V. O. Volochnyuk, D. M.; Irgashev, R. A.; Sosnovskikh, V. Y. Synthesis 2009 3869-3879; (c) Iaroshenko, V. O.; Mkrtchyan, S.; Ghazaryan, G.; Hakobyan, A.; Maalik, A.; Supe, L.; Villinger, A.; Tolmachev, A.; Ostrovskyi, D.; Sosnovskikh, V. Y.; Ghochikyan, T. V.; Langer, P. Synthesis 2011, 469-479; (d) Mkrtchyan, S.; Iaroshenko, V. O.; Dudkin, S.; Gevorgyan, A.; Vilches-Herrera, M.; Ghazaryan, G.; Volochnyuk, D.; Ostrovskyi, D.; Ahmed, Z.; Villinger, A.; Sosnovskikh, V. Y.; Langer, P. Org. Biomol. Chem. 2010, 8, 5280-5284; (e) Iaroshenko, V. O. Mkrtchyan, S.; Gevorgyan, A.; Vilches-Herrera, M.; Sevenard, D. V.; Villinger, A.; Ghochikyan, T. V.; Saghiyan, A.; Sosnovskikh, V. Y.; Langer, P. Tetrahedron 2012 2532-2543; (f) Iaroshenko, V. O.; Mkrtchyan, S.; Gevorgyan, A.; Miliutina, M.; Villinger, A.; Volochnyuk, D.; Sosnovskikh, V. Y.; Langer, P. Org. Biomol. Chem. 2012, 10, 890-894; (g) Iaroshenko, V. O.; Wang, Y.; Zhang, B.; Volochnyuk, D.; Sosnovskikh, V. Ya. Synthesis 2009, 2393-2402; (h) Iaroshenko, V. O.; Ostrovskyi, D.; Petrosyan, A.; Mkrtchyan, S.; Villinger, A.; Langer, P. Journal of Organic Chemistry 2011, 2899-2903; (i) Fatunsin, O; Iaroshenko, V. O; Dudkin, S.; Mkrtchyan, S.; Villinger, A.; Langer, P. Tetrahedron Letters 2010, 51 4693-4695; (j) Iaroshenko, V. O.; Specowius, V.; Vlach, K.; Vilches-Herrera, M.; Ostrovskyi, D.; Mkrtchyan, S.; Villinger, A.; Langer, P. Tetrahedron 2011, 67, 5663-5677; (k) Iaroshenko, V. O.; Bunescu, A.; Spannenberg, A.; Langer, P. Chemistry - A European Journal 2011, 17, 7188-7192; (1) Iaroshenko, V. O.; Mkrtchyan, S.; Villinger, A. Synthesis 2013, 205-218; (m) Iaroshenko, V. O.; Vilches-Herrera, M.; Gevorgyan, A.; Arakelyan, K.; Ostrovskyi, D.; Abbasi, M. S. A. Mkrtchyan, S.; Supe, L.; Hakobyan, A.; Villinger, A.; Volochnyuk, D. M.; Tolmachev, A. Tetrahedron 2013, 69, 1217-1228; (n) Iaroshenko, V. O.; Ali, I.; Mkrtchyan, S.; Semeniuchenko, V.; Ostrovskyi, D.; Langer, P. Synlett 2012, 18, 2603-2605.

5. Iaroshenko, V. O.; Knepper, I.; Zahid, M.; Kuzora, R.; Dudkin, S.; Villinger, A.; Langer, P. Org. Biomol. Chem. 2012, 10, 2955-2959.

6. Vijavalakshmi, S.; Ragunath, L.; Rajendran, S. P. Heterocycl. Commun. 2001, 7, 177-182.

7. Salman, G. A.; Hussain, M.; Iaroshenko, V.; Villinger, A.; Langer, P. Adv. Synth. Catal. 2011, 353, 331-336.

8. (a) Lin, C.-F.; Lu, W.-D.; Wang, I.-W.; Wu, M.-J. Synlett 2003, 2057-2061; (b) Waldo, J. P.; Larock, R. C. J. Org. Chem. 2007, 72, 9643-9647.

9. Baruah, B.; Bhuyan, P. J. Tetrahedron 2009, 65, 7099-7104

10. (a) Bernini, R.; Cacchi, S.; Fabrizi, G.; Sferrazza, A. Synthesis 2009, 1209-1219; (b) Zhao, T.; Xu, B. Org. Lett. 2010, 12, 212-215.

11. Crystallographic data (excluding structure factors) for the structures $\mathbf{3 f}$, and $\mathbf{6 i}$, reported in this paper have been deposited with the Cambridge Crystallographic Data Centre as supplementary publication nos. 919223 \& 919224 and can be obtained free of charge on application to CCDC, 12 Union Road, Cambridge CB2 1EZ, UK; fax: +44 1223 336033; e-mail: deposit@ccdc.cam.ac.uk, or via www.ccdc.cam.ac.uk/data_request/cif.

12. Mahesh, R.; Perumal, R. V.; Pandi, P. V. Biol. Pharm. Bull. 2004, 27, 1403-1405. 